\title{
Switching between novel samarium(II)-mediated cyclizations by a simple change in alcohol cosolvent
}

Thomas K. Hutton, Kenneth W. Muir and David J. Procter*

Department of Chemistry, The Joseph Black Building, University of Glasgow, Glasgow, G12 8QQ.

davidp@chem.gla.ac.uk

\section{Contents}

S2-S29 Experimental

S30-S62 $\quad{ }^{1} \mathrm{H}$ and ${ }^{13} \mathrm{C}-\mathrm{NMR}$ spectra of new compounds

S63-S81 X-ray Crystallographic data for 5, 15, 35 


\section{Experimental}

General Experimental Considerations have been described previously. ${ }^{1}$

\section{General Samarium(II) Cyclisation Procedure A}

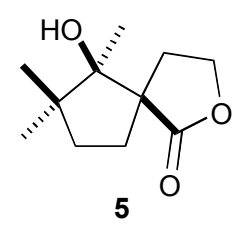

rel-(5R, 6R)-6-hydroxy-6,7,7-trimethyl-2-oxa-spiro[4.4]nonan-1-one 5

Dry $\mathrm{MeOH}(1.4 \mathrm{ml})$ was added to a stirred solution of $\mathrm{SmI}_{2}(0.1 \mathrm{M}$ in THF, $5.10 \mathrm{ml}$, $0.510 \mathrm{mmol}, 4 \mathrm{eq})$ at $0^{\circ} \mathrm{C}$ and the resulting solution was stirred for $10 \mathrm{~min}$. (E)-3-(3,3Dimethyl-4-oxo-pentylidene)-dihydro-furan-2-one 4 (25 mg, $0.127 \mathrm{mmol}, 1 \mathrm{eq}$ ), in THF $(0.5 \mathrm{ml})$ was added, and the resultant solution stirred at $0^{\circ} \mathrm{C}$ for $2 \mathrm{~h}$. The reaction was quenched by opening to the air, followed by the addition of aqueous saturated $\mathrm{NaCl}(10 \mathrm{ml})$. The aqueous layer was separated and extracted with $80 \%$ EtOAc in petroleum ether $\left(40-60^{\circ} \mathrm{C}\right)(4 \times 15 \mathrm{ml})$. The combined organic extracts were dried $\left(\mathrm{MgSO}_{4}\right)$, and concentrated in vacuo to give the crude product. Purification by column chromatography (eluting with $40 \%$ EtOAc in petroleum ether $\left(40-60^{\circ} \mathrm{C}\right)$ ), gave rel(5R, 6R)-6-hydroxy-6,7,7-trimethyl-2-oxa-spiro[4.4]nonan-1-one 5 (15.8 mg, 0.08 mmol, 63\%) as a white crystalline solid: $\mathrm{mp} 87-90^{\circ} \mathrm{C}(20 \%$ EtOAc in petroleum ether $\left.\left(40-60^{\circ} \mathrm{C}\right)\right): \square_{\max }($ neat $) / \mathrm{cm}^{-1} 3431 \mathrm{~s}(\mathrm{OH}), 1733 \mathrm{~s}(\mathrm{C}=\mathrm{O}), 1471 \mathrm{~m}, 1376 \mathrm{~m}, 1221 \mathrm{~m}$, $1199 \mathrm{~m}$ and 1026m; $\square_{\mathrm{H}}\left(400 \mathrm{MHz}, \mathrm{CDCl}_{3}\right) 0.87\left(3 \mathrm{H}, \mathrm{s}, \mathrm{CH}_{3}\right), 1.02\left(3 \mathrm{H}, \mathrm{s}, \mathrm{CH}_{3}\right), 1.10$ $\left(3 \mathrm{H}, \mathrm{s}, \mathrm{CH}_{3}\right), 1.52-1.59\left(1 \mathrm{H}, \mathrm{m}, 1 \mathrm{H}\right.$ from $\left.\mathrm{C}\left(\mathrm{CH}_{3}\right)_{2} \mathrm{CH}_{2}\right), 1.66-1.72(1 \mathrm{H}, \mathrm{m}, 1 \mathrm{H}$ from $\left.\mathrm{C}\left(\mathrm{CH}_{3}\right)_{2} \mathrm{CH}_{2} \mathrm{CH}_{2}\right), 1.84-1.91\left(1 \mathrm{H}, \mathrm{m}, 1 \mathrm{H}\right.$ from $\left.\mathrm{C}\left(\mathrm{CH}_{3}\right)_{2} \mathrm{CH}_{2}\right), 2.02-2.07(1 \mathrm{H}, \mathrm{m}, 1 \mathrm{H}$ from $\left.\mathrm{OCH}_{2} \mathrm{CH}_{2}\right), 2.15-2.23\left(1 \mathrm{H}, \mathrm{m}, 1 \mathrm{H}\right.$ from $\left.\mathrm{C}\left(\mathrm{CH}_{3}\right)_{2} \mathrm{CH}_{2} \mathrm{CH}_{2}\right), 2.34-2.42(1 \mathrm{H}, \mathrm{m}$, $1 \mathrm{H}$ from $\left.\mathrm{OCH}_{2} \mathrm{CH}_{2}\right), 4.09-4.15\left(1 \mathrm{H}, \mathrm{m}, 1 \mathrm{H}\right.$ from $\left.\mathrm{OCH}_{2}\right), 4.23-4.28(1 \mathrm{H}, \mathrm{m}, 1 \mathrm{H}$ from $\left.\mathrm{OCH}_{2}\right)$ and $4.65(1 \mathrm{H}, \mathrm{s}, \mathrm{OH})$; $\square_{\mathrm{C}}\left(100 \mathrm{MHz}, \mathrm{CDCl}_{3}\right) 19.3\left(\mathrm{CH}_{3}\right), 24.0\left(\mathrm{CH}_{3}\right), 27.6$ $\left.\left(\mathrm{CH}_{3}\right), 33.2\left(\mathrm{C}\left(\mathrm{CH}_{3}\right)_{2} \mathrm{CH}_{2} \mathrm{CH}_{2}\right), 35.1\left(\mathrm{OCH}_{2} \mathrm{CH}_{2}\right), 38.7,\left(\mathrm{C}\left(\mathrm{CH}_{3}\right)_{2} \mathrm{CH}_{2}\right), 46.0\left(\mathrm{C}_{\left(\mathrm{CH}_{3}\right.}\right)_{2}\right)$,

\footnotetext{
${ }^{1}$ Edmonds, D. J.; Muir, K. W.; Procter, D. J. J. Org. Chem. 2003, 68, 3190.
} 
$55.2(\mathrm{CC}(\mathrm{O})), 65.8\left(\mathrm{OCH}_{2}\right), 84.8\left(\mathrm{C}(\mathrm{OH}) \mathrm{CH}_{3}\right)$ and $183.3(\mathrm{C}(\mathrm{O})) ; \mathrm{m} / z(\mathrm{EI}$ mode $) 198$ (( $\left.\mathrm{M}^{+\bullet}\right)$ 10\%), 180 (25), 170 (20), 153 (40), 138 (25), 128 (95), 112 (100), 99 (100), 85 (100), 83 (100), 55 (25) and 43 (55); (Found: C, 66.82; $\mathrm{H}, 9.35 ; \mathrm{C}_{11} \mathrm{H}_{18} \mathrm{O}_{3}$ requires $\mathrm{C}$, $66.64 ; \mathrm{H}, 9.15)$.

\section{General Oxidation/Olefination Procedure B}

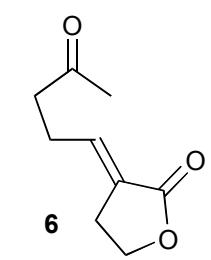

\section{(E)-3-(4-Oxo-pentylidene)-dihydro-furan-2-one 6}

DMSO (681 $\square 1,9.60 \mathrm{mmol}, 4 \mathrm{eq})$ was added to a stirred solution of $(\mathrm{COCl})_{2}(419 \square 1$, $4.80 \mathrm{mmol}, 2 \mathrm{eq})$ in $\mathrm{CH}_{2} \mathrm{Cl}_{2}(10 \mathrm{ml})$ at $-78^{\circ} \mathrm{C}$ and the resulting solution was stirred for 10 min. 1,4-Pentanediol (250 mg, $2.40 \mathrm{mmol}, 1 \mathrm{eq}$ ) was then added as a solution in $\mathrm{CH}_{2} \mathrm{Cl}_{2}(10 \mathrm{ml})$ and the resultant solution stirred for a further $30 \mathrm{~min}$. Triethylamine (3.44 $\mathrm{ml}, 24.0 \mathrm{mmol}, 10 \mathrm{eq})$ was then added. After $2 \mathrm{~h}$ (1butyrolactonylidene)triphenylphosphorane (1.66 $\mathrm{g}, 4.80 \mathrm{mmol}, 2 \mathrm{eq})$ was added as a solution in $\mathrm{CH}_{2} \mathrm{Cl}_{2}(20 \mathrm{ml})$ and the reaction mixture allowed to warm to room temperature and stirred for $16 \mathrm{~h}$. Aqueous saturated $\mathrm{NaHCO}_{3}(10 \mathrm{ml})$ was then added, and the aqueous layer was separated and extracted with $80 \%$ EtOAc in petroleum ether $\left(40-60^{\circ} \mathrm{C}\right)(4 \times 20 \mathrm{ml})$. The combined organic extracts were dried $\left(\mathrm{MgSO}_{4}\right)$, and concentrated in vacuo to give the crude product. Purification by column chromatography (eluting with $25 \%$ EtOAc in petroleum ether $\left(40-60^{\circ} \mathrm{C}\right)$ ), gave $(E)-3$ (4-oxo-pentylidene)-dihydro-furan-2-one 6 (399 $\mathrm{mg}, 2.37 \mathrm{mmol}, 99 \%$ ) as a yellow oil: $\square_{\max }\left(\right.$ neat) $/ \mathrm{cm}^{-1} 1739 \mathrm{~s}$ (lactone $\left.\mathrm{C}=\mathrm{O}\right), 1716$ s (ketone $\left.\mathrm{C}=\mathrm{O}\right), 1681 \mathrm{~s}(\mathrm{C}=\mathrm{C}), 1405 \mathrm{~m}$, $1383 \mathrm{~m}, 1238 \mathrm{~m}, 1190 \mathrm{~m}, 1038 \mathrm{~m}, 1021 \mathrm{~m}$ and $997 \mathrm{~m}$; $\square_{\mathrm{H}}\left(400 \mathrm{MHz}, \mathrm{CDCl}_{3}\right) 2.19(3 \mathrm{H}, \mathrm{s}$, $\left.\mathrm{C}(\mathrm{O}) \mathrm{CH}_{3}\right), 2.46\left(2 \mathrm{H}\right.$, apparent q, J 7.1, $\left.\mathrm{CH}_{2} \mathrm{CH}=\mathrm{C}\right), 2.67\left(2 \mathrm{H}, \mathrm{t}, J 7.1 \mathrm{CH}_{2} \mathrm{C}(\mathrm{O})\right), 2.96$ $\left(2 \mathrm{H}, \mathrm{dt}, J 2.9,7.5, \mathrm{CH}_{2} \mathrm{CH}_{2} \mathrm{O}\right), 4.41\left(2 \mathrm{H}, \mathrm{t}, J\right.$ 7.5, $\left.\mathrm{CH}_{2} \mathrm{O}\right)$ and $6.64(1 \mathrm{H}, \mathrm{tt}, J$ 2.9, 7.5, $\mathrm{CH}=\mathrm{C}) ; \square_{\mathrm{C}}\left(100 \mathrm{MHz}, \mathrm{CDCl}_{3}\right) 24.5\left(\mathrm{CH}_{2} \mathrm{CH}=\mathrm{C}\right), 25.5\left(\mathrm{CH}=\mathrm{CCH}_{2}\right), 30.4\left(\mathrm{C}(\mathrm{O}) \mathrm{CH}_{3}\right)$, $41.8\left(\mathrm{CH}_{2} \mathrm{C}(\mathrm{O})\right), 65.9\left(\mathrm{CH}_{2} \mathrm{O}\right), 126.9(\mathrm{CH}=C), 138.9(\mathrm{CH}=\mathrm{C}), 171.5(C(\mathrm{O}) \mathrm{O})$ and 
$207.0(C(\mathrm{O})) ; \mathrm{m} / \mathrm{z}\left(\right.$ EI mode) $168\left(\left(\mathrm{M}^{+\bullet}\right)\right.$ 10\%), $125(60), 111(5), 83$ (100), $79(15)$ and 47 (20); (Found: $\left(\mathrm{M}^{+\bullet}\right), 168.0785 . \mathrm{C}_{9} \mathrm{H}_{12} \mathrm{O}_{3}$ requires $\left.M, 168.0786\right)$.

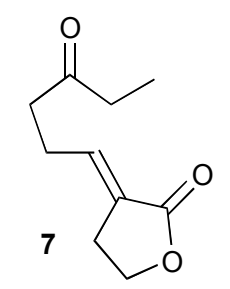

\section{(E)-3-(4-Oxo-hexylidene)-dihydro-furan-2-one 7}

As for general procedure B. Hexane-1,4-diol (180 mg, $1.52 \mathrm{mmol}, 1 \mathrm{eq}$ ), after oxidation and reaction with (1-butyrolactonylidene)triphenylphosphorane (1.05 g, $3.04 \mathrm{mmol}, 2 \mathrm{eq}$ ) for $16 \mathrm{~h}$ and purification of the crude product mixture by column chromatography (eluting with $80 \%$ EtOAc in petroleum ether $\left(40-60^{\circ} \mathrm{C}\right)$ ), gave $(E)-3$ (4-oxo-hexylidene)-dihydro-furan-2-one $7(115 \mathrm{mg}, 0.63 \mathrm{mmol}, 42 \%)$ as a pale yellow oil: $\square_{\max }\left(\right.$ neat) $/ \mathrm{cm}^{-1} 1747$ s $($ ester $\mathrm{C}=\mathrm{O}), 1709$ s (ketone $\mathrm{C}=\mathrm{O}$ ), 1679s $(\mathrm{C}=\mathrm{C})$, $1374 \mathrm{~m}, 1352 \mathrm{~m}, 1211 \mathrm{~m}$ and $1022 \mathrm{~m}$; $\square_{\mathrm{H}}\left(400 \mathrm{MHz}, \mathrm{CDCl}_{3}\right) 1.08\left(3 \mathrm{H}, \mathrm{t}, J 7.4, \mathrm{CH}_{3}\right)$, $2.43-2.50\left(4 \mathrm{H}, \mathrm{m}, 2 \mathrm{H}\right.$ from $\mathrm{CH}_{2} \mathrm{CH}_{3}, 2 \mathrm{H}$ from $\left.\mathrm{CH}_{2} \mathrm{CH}=\mathrm{C}\right), 2.64(2 \mathrm{H}, \mathrm{t}, J$ 7.1, $\left.\mathrm{CH}_{2} \mathrm{C}(\mathrm{O})\right), 2.93\left(2 \mathrm{H}, \mathrm{dt}, J 3.0,7.5, \mathrm{CH}_{2} \mathrm{CH}_{2} \mathrm{O}\right), 4.40\left(2 \mathrm{H}, \mathrm{t}, J 7.5, \mathrm{CH}_{2} \mathrm{O}\right)$ and 6.63 $(1 \mathrm{H}, \mathrm{tt}, J 3.0,7.6, \mathrm{CH}=\mathrm{C})$; $\square_{\mathrm{C}}\left(100 \mathrm{MHz}, \mathrm{CDCl}_{3}\right) 8.1\left(\mathrm{CH}_{3}\right), 24.5\left(\mathrm{CH}_{2} \mathrm{CH}=\mathrm{C}\right), 25.5$ $\left(\mathrm{CH}_{2} \mathrm{C}=\mathrm{CH}\right), 36.4\left(\mathrm{CH}_{2} \mathrm{CH}_{3}\right), 40.5\left(\mathrm{CH}_{2} \mathrm{C}(\mathrm{O})\right), 65.9\left(\mathrm{CH}_{2} \mathrm{O}\right), 126.8(\mathrm{CH}=C), 139.1$ $(C \mathrm{H}=\mathrm{C}), 171.6(C(\mathrm{O}) \mathrm{O})$ and $209.9(C(\mathrm{O})) ; \mathrm{m} / \mathrm{z}(\mathrm{EI}$ mode $) 182\left(\left(\mathrm{M}^{+\bullet}\right) 45 \%\right), 153(10)$, 125 (100), 109 (15), 97 (15), 81 (50), 57 (65) and 53 (20); (Found: ( $\mathrm{M}^{+\bullet}$ ), 182.0942. $\mathrm{C}_{10} \mathrm{H}_{14} \mathrm{O}_{3}$ requires $\left.M, 182.0943\right)$.

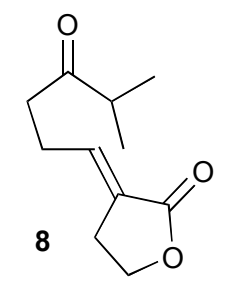




\section{(E)-3-(5-Methyl-4-oxo-hexylidene)-dihydro-furan-2-one 8}

As for general procedure B. 5-Methyl-hexane-1,4-diol (200 mg, $1.51 \mathrm{mmol}, 1 \mathrm{eq}$ ), after oxidation and reaction with (1-butyrolactonylidene)triphenylphosphorane (1.05 $\mathrm{g}, 3.03 \mathrm{mmol}, 2 \mathrm{eq}$ ) for $16 \mathrm{~h}$ and purification of the crude product mixture by column chromatography (eluting with $80 \%$ EtOAc in petroleum ether $\left(40-60^{\circ} \mathrm{C}\right)$ ), gave $(E)-3$ (5-methyl-4-oxo-hexylidene)-dihydro-furan-2-one 8 (196 mg, $1.00 \mathrm{mmol}, 66 \%$ ) as a pale yellow oil: $\square_{\max }$ (neat)/cm $\mathrm{cm}^{-1} 2970 \mathrm{w}, 1749$ s (ester $\mathrm{C}=\mathrm{O}$ ), 1707s (ketone $\mathrm{C}=\mathrm{O}$ ), 1680s $(\mathrm{C}=\mathrm{C}), 1468 \mathrm{~m}, 1381 \mathrm{~m}, 1215 \mathrm{~m}, 1194 \mathrm{~m}$ and $1026 \mathrm{~m}$; $\square_{\mathrm{H}}\left(400 \mathrm{MHz}, \mathrm{CDCl}_{3}\right) 1.11$ $\left(3 \mathrm{H}, \mathrm{d}, J 7.0, \mathrm{CH}\left(\mathrm{CH}_{3}\right)_{2}\right), 1.45\left(3 \mathrm{H}, \mathrm{d}, J 7.0, \mathrm{CH}\left(\mathrm{CH}_{3}\right)_{2}\right), 2.47(2 \mathrm{H}$, apparent q, $J$ 8.8, $\left.\mathrm{CH}_{2} \mathrm{CH}=\mathrm{C}\right), 2.65\left(1 \mathrm{H}\right.$, septet, $J$ 7.0, $\left.\mathrm{CH}\left(\mathrm{CH}_{3}\right)_{2}\right), 2.79\left(2 \mathrm{H}, \mathrm{t}, J 6.4, \mathrm{CH}_{2} \mathrm{C}(\mathrm{O})\right), 2.94-$ $3.00\left(2 \mathrm{H}, \mathrm{m}, \mathrm{CH}_{2} \mathrm{CH}_{2} \mathrm{O}\right), 4.40\left(2 \mathrm{H}, \mathrm{t}, J\right.$ 7.4, $\left.\mathrm{CH}_{2} \mathrm{O}\right)$ and $6.63(1 \mathrm{H}, \mathrm{tt}, J$ 2.9, 7.6, $\mathrm{CH}=\mathrm{C})$; $\mathrm{C}_{\mathrm{c}}\left(100 \mathrm{MHz}, \mathrm{CDCl}_{3}\right) 18.6\left(\mathrm{CH}\left(\mathrm{CH}_{3}\right)_{2}\right), 18.7\left(\mathrm{CH}\left(\mathrm{CH}_{3}\right)_{2}\right), 24.6\left(\mathrm{CH}_{2} \mathrm{CH}=\mathrm{C}\right)$, $25.5\left(\mathrm{CH}_{2} \mathrm{CH}_{2} \mathrm{O}\right), 38.5\left(\mathrm{CH}_{2} \mathrm{C}(\mathrm{O})\right), 41.3\left(\mathrm{CH}\left(\mathrm{CH}_{3}\right)_{2}\right), 65.9\left(\mathrm{CH}_{2} \mathrm{O}\right), 122.6(\mathrm{CH}=C)$, $139.3(C \mathrm{H}=\mathrm{C}), 171.6(C(\mathrm{O}) \mathrm{O})$ and $213.2(C(\mathrm{O})) ; \mathrm{m} / \mathrm{z}(\mathrm{EI}$ mode $) 196\left(\left(\mathrm{M}^{+\bullet}\right) 35 \%\right), 153$ (25), 125 (100), 109 (30), 81 (50), 79 (30) and 43 (50); (Found: (M+•), 196.1100. $\mathrm{C}_{11} \mathrm{H}_{16} \mathrm{O}_{3}$ requires $\left.M, 196.1099\right)$.

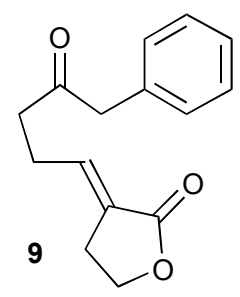

\section{(E)-3-(4-Oxo-5-phenyl-pentylidene)-dihydro-furan-2-one 9}

As for general procedure B. 5-Phenyl-pentane-1,4-diol (100 mg, $0.56 \mathrm{mmol}, 1 \mathrm{eq}$ ), after oxidation and reaction with (1-butyrolactonylidene)triphenylphosphorane (385 $\mathrm{mg}, 1.11 \mathrm{mmol}, 2 \mathrm{eq}$ ) for $16 \mathrm{~h}$ and purification of the crude product mixture by column chromatography (eluting with $40 \%$ EtOAc in petroleum ether $\left(40-60^{\circ} \mathrm{C}\right)$ ), gave (E)-3-(4-oxo-5-phenyl-pentylidene)-dihydro-furan-2-one 9 (78 mg, $0.34 \mathrm{mmol}$, $61 \%$ ) as a yellow oil: $\square_{\max }\left(\right.$ neat) $/ \mathrm{cm}^{-1} 2917 \mathrm{~s}, 1751 \mathrm{~s}$ (lactone $\mathrm{C}=\mathrm{O}$ ), $1717 \mathrm{~s}$ (ketone $\mathrm{C}=\mathrm{O}), 1681 \mathrm{~s}(\mathrm{C}=\mathrm{C}), 1497 \mathrm{~m}, 1454 \mathrm{~m}, 1438 \mathrm{~m}, 1412 \mathrm{~m}, 1382 \mathrm{~m}, 1360 \mathrm{~m}, 1194 \mathrm{~m}, 1030 \mathrm{~m}$ and 961m; $\square_{\mathrm{H}}\left(400 \mathrm{MHz}, \mathrm{CDCl}_{3}\right) 2.43\left(2 \mathrm{H}\right.$, apparent q, $\left.J 7.1, \mathrm{CH}_{2} \mathrm{CH}=\mathrm{C}\right), 2.67(2 \mathrm{H}, \mathrm{t}$, 
J 7.1 $\left.\mathrm{CH}_{2} \mathrm{C}(\mathrm{O})\right), 2.91\left(2 \mathrm{H}, \mathrm{dt}, J 3.0,7.4, \mathrm{CH}_{2} \mathrm{C}=\mathrm{CH}\right), 3.72\left(2 \mathrm{H}, \mathrm{s}, \mathrm{CH}_{2} \mathrm{Ph}\right), 4.38(2 \mathrm{H}, \mathrm{t}$, J 7.4, $\left.\mathrm{CH}_{2} \mathrm{O}\right), 6.57(1 \mathrm{H}, \mathrm{tt}, J 3.0,7.4, \mathrm{CH}=\mathrm{C}), 7.20-7.22(1 \mathrm{H}, \mathrm{m}, \mathrm{Ar} \mathrm{CH}), 7.26-7.32$ $(2 \mathrm{H}, \mathrm{m}, 2 \times \mathrm{Ar} \mathrm{CH})$ and $7.34-7.38(2 \mathrm{H}, \mathrm{m}, 2 \mathrm{x} \mathrm{Ar} \mathrm{CH})$; $\square_{\mathrm{c}}\left(100 \mathrm{MHz}, \mathrm{CDCl}_{3}\right) 24.5$ $\left(\mathrm{CH}_{2} \mathrm{CH}=\mathrm{C}\right), 25.4\left(\mathrm{CH}_{2} \mathrm{C}=\mathrm{CH}\right), 40.2\left(\mathrm{CH}_{2} \mathrm{C}(\mathrm{O})\right), 50.7\left(\mathrm{CH}_{2} \mathrm{Ph}\right), 65.8\left(\mathrm{CH}_{2} \mathrm{O}\right), 127.0$ $(\mathrm{CH}=C), 127.7(\operatorname{Ar} C \mathrm{H}), 129.3(2 \times \mathrm{Ar} C \mathrm{H}), 129.7$ (2 x Ar $\mathrm{CH}), 134.2(\operatorname{Ar} C), 138.8$ $(C \mathrm{H}=\mathrm{C}), 171.5(C(\mathrm{O}) \mathrm{O})$ and $206.9(C(\mathrm{O})) ; \mathrm{m} / \mathrm{z}\left(\mathrm{EI}\right.$ mode) $244\left(\left(\mathrm{M}^{+\bullet}\right) 37 \%\right), 223(5)$, 205 (2), 176 (5), 153 (100), 125 (45), 119 (65), 91 (100), 81 (50), 65 (40) and 41 (22); (Found: $\left(\mathrm{M}^{+\bullet}\right), 244.1099 . \mathrm{C}_{15} \mathrm{H}_{16} \mathrm{O}_{3}$ requires $\left.M, 244.1099\right)$.

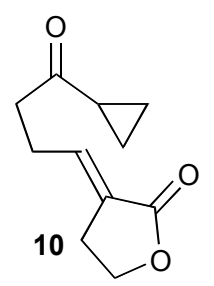

(E)-3-(4-Oxo-4-cyclopropyl-butylidene)-dihydro-furan-2-one 10

As for general procedure B. 1-Cyclopropyl-butane-1,4-diol (408 mg, $2.45 \mathrm{mmol}, 1$ eq), after oxidation and reaction with (1-butyrolactonylidene)triphenylphosphorane $(1.70 \mathrm{~g}, 4.92 \mathrm{mmol}, 2 \mathrm{eq})$ for $16 \mathrm{~h}$ and purification of the crude product mixture by column chromatography (eluting with $50 \%$ EtOAc in petroleum ether $\left(40-60^{\circ} \mathrm{C}\right)$ ), gave (E)-3-(4-oxo-4-cyclopropyl-butylidene)-dihydro-furan-2-one 10 (111 mg, 0.57 mmol, $48 \%)$ as a white crystalline solid: $\mathrm{mp} 67-69^{\circ} \mathrm{C}\left(20 \% \mathrm{Et}_{2} \mathrm{O}\right.$ in petroleum ether (30-40 $\left.{ }^{\circ} \mathrm{C}\right): \square_{\max }\left(\right.$ neat) $/ \mathrm{cm}^{-1} 2951 \mathrm{~m}, 2899 \mathrm{~m}, 1738$ s (lactone $\mathrm{C}=\mathrm{O}$ ), 1693s (ketone $\mathrm{C}=\mathrm{O}), 1678 \mathrm{~s}(\mathrm{C}=\mathrm{C}), 1496 \mathrm{~m}, 1311 \mathrm{~m}$ and $815 \mathrm{~m}$; $\square_{\mathrm{H}}\left(400 \mathrm{MHz}, \mathrm{CDCl}_{3}\right) 0.79 \square 0.85$ $\left(2 \mathrm{H}, \mathrm{m}, \mathrm{CH}(\mathrm{OH}) \mathrm{CHCH}_{2}{ }^{\mathrm{A}}\right), 0.94 \square 0.98\left(2 \mathrm{H}, \mathrm{m}, \mathrm{CH}(\mathrm{OH}) \mathrm{CHCH}_{2}{ }^{\mathrm{B}}\right), 1.78 \square 1.90(1 \mathrm{H}$, m, $\mathrm{CH}(\mathrm{OH}) \mathrm{CH}), 2.40\left(2 \mathrm{H}\right.$, apparent q, $J$ 7.3, $\left.\mathrm{CH}_{2} \mathrm{CH}=\mathrm{C}\right), 2.72(2 \mathrm{H}, \mathrm{t}, J$ 7.3, $\left.\mathrm{CH}_{2} \mathrm{C}(\mathrm{O})\right), 2.86\left(2 \mathrm{H}\right.$, apparent q, J 7.4, $\left.\mathrm{CH}_{2} \mathrm{C}=\mathrm{CH}\right), 4.30\left(2 \mathrm{H}, \mathrm{t}, J 7.4, \mathrm{CH}_{2} \mathrm{O}\right)$ and 6.60 $(1 \mathrm{H}, \mathrm{tt}, J$ 1.6, 7.3, $\mathrm{CH}=\mathrm{C})$; $\square_{\mathrm{C}}\left(100 \mathrm{MHz}, \mathrm{CDCl}_{3}\right) 9.3\left(\mathrm{C}(\mathrm{OH}) \mathrm{CHCH}_{2}{ }^{\mathrm{A}}\right), 9.4$ $\left(\mathrm{C}(\mathrm{OH}) \mathrm{CHCH}_{2}{ }^{\mathrm{B}}\right), 19.0(\mathrm{CH}(\mathrm{OH}) \mathrm{CH}), 22.7\left(\mathrm{CH}_{2} \mathrm{CH}=\mathrm{C}\right), 23.5\left(\mathrm{CH}_{2} \mathrm{C}=\mathrm{CH}\right), 39.6$ $\left(\mathrm{CH}_{2} \mathrm{C}(\mathrm{O})\right), 63.9\left(\mathrm{CH}_{2} \mathrm{O}\right), 124.7(\mathrm{CH}=\mathrm{C}), 137.3(\mathrm{CH}=\mathrm{C}), 169.6(\mathrm{C}(\mathrm{O}) \mathrm{O})$ and 207.3 $(C(\mathrm{O})) ; m / z$ (EI mode) $194\left(\left(\mathrm{M}^{+\bullet}\right), 28 \%\right), 125$ (100), 85 (50), 83 (76), 69 (83), 47 (16) and 41 (39); (Found: $\left(\mathrm{M}^{+\bullet}\right)$ 194.0942. $\mathrm{C}_{11} \mathrm{H}_{14} \mathrm{O}_{3}$ requires $M$, 194.0943). 


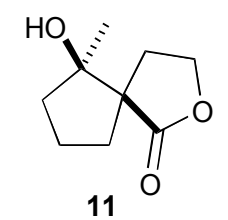

\section{rel-(5R, 6R)-6-Hydroxy-6-methyl-2-oxa-spiro[4.4]nonan-1-one $11^{2}$}

As for general procedure A. (E)-3-(4-Oxo-pentylidene)-dihydro-furan-2-one 6 (50 $\mathrm{mg}, 0.30 \mathrm{mmol}, 1 \mathrm{eq}$ ), after a reaction time of $2 \mathrm{~h}$ and purification of the crude product mixture by column chromatography (eluting with $50 \%$ EtOAc in petroleum ether $\left.\left(40-60^{\circ} \mathrm{C}\right)\right)$, gave rel-(5R, 6R)-6-hydroxy-6-methyl-2-oxa-spiro[4.4]nonan-1-one 11 (36 mg, $0.21 \mathrm{mmol}, 71 \%)$ as a clear, colourless oil: $\square_{\max }($ neat $) / \mathrm{cm}^{-1} 3480 \mathrm{bs}(\mathrm{OH})$, 2971s, 2874s, 1758s (C=O), 1450m, 1376s, 1302w, 1200m, 1149m, 1103m, 1030m, $1001 \mathrm{~m}, 958 \mathrm{~m}$ and $937 \mathrm{~m}$; $\mathrm{\square}_{\mathrm{H}}\left(400 \mathrm{MHz}, \mathrm{CDCl}_{3}\right) 1.23\left(3 \mathrm{H}, \mathrm{s}, \mathrm{C}(\mathrm{OH}) \mathrm{CH}_{3}\right), 1.58-1.75$ (3H, m, $1 \mathrm{H}$ from $\mathrm{CH}_{2} \mathrm{CH}_{2} \mathrm{CH}_{2} \mathrm{C}(\mathrm{OH}), 1 \mathrm{H}$ from $\mathrm{CH}_{2} \mathrm{CH}_{2} \mathrm{C}(\mathrm{OH}), 1 \mathrm{H}$ from $\mathrm{CH}_{2} \mathrm{C}(\mathrm{OH})$ ), $1.88\left(1 \mathrm{H}\right.$, ddd, $J 3.0,6.7,12.7,1 \mathrm{H}$ from $\left.\mathrm{CH}_{2} \mathrm{CH}_{2} \mathrm{O}\right), 1.92-1.99(2 \mathrm{H}, \mathrm{m}, 1 \mathrm{H}$ from $\mathrm{CH}_{2} \mathrm{CH}_{2} \mathrm{CH}_{2} \mathrm{C}(\mathrm{OH}), 1 \mathrm{H}$ from $\left.\mathrm{CH}_{2} \mathrm{CH}_{2} \mathrm{C}(\mathrm{OH})\right), 2.22-2.31$ (2H, m, $1 \mathrm{H}$ from $\mathrm{CH}_{2} \mathrm{C}(\mathrm{OH}), 1 \mathrm{H}$ from $\left.\mathrm{CH}_{2} \mathrm{CH}_{2} \mathrm{O}\right), 3.60(1 \mathrm{H}, \mathrm{s}, \mathrm{OH}), 4.15(1 \mathrm{H}, \mathrm{dt}, J 6.7,9.0,1 \mathrm{H}$ from $\left.\mathrm{CH}_{2} \mathrm{O}\right)$ and $4.27\left(1 \mathrm{H}, \mathrm{dt}, J 3.0,9.0,1 \mathrm{H}\right.$ from $\left.\mathrm{CH}_{2} \mathrm{O}\right) ; \square_{\mathrm{c}}\left(100 \mathrm{MHz}, \mathrm{CDCl}_{3}\right) 20.7$ $\left(\mathrm{CH}_{2} \mathrm{CH}_{2} \mathrm{C}(\mathrm{OH})\right), 23.3\left(\mathrm{CH}_{3}\right), 32.4 \quad\left(\mathrm{C} \mathrm{H}_{2} \mathrm{CH}_{2} \mathrm{O}\right), 34.1 \quad\left(C \mathrm{H}_{2} \mathrm{C}(\mathrm{OH})\right), \quad 39.0$ $\left(\mathrm{CH}_{2} \mathrm{CH}_{2} \mathrm{CH}_{2} \mathrm{C}(\mathrm{OH})\right), 55.0(\mathrm{CC}(\mathrm{O})), 65.8\left(\mathrm{CH}_{2} \mathrm{O}\right), 82.2(\mathrm{C}(\mathrm{OH}))$ and $182.0(\mathrm{C}(\mathrm{O}))$; m/z (EI mode) $170\left(\left(\mathrm{M}^{+\bullet}\right)\right.$ 7\%), 155 (2), 128 (7), 112 (40), 99 (100), 72 (25), 53 (10) and 43 (35); (Found: $\left(\mathrm{M}^{+\bullet}\right), 170.0942 . \mathrm{C}_{9} \mathrm{H}_{14} \mathrm{O}_{3}$ requires $\left.M, 170.0943\right)$.

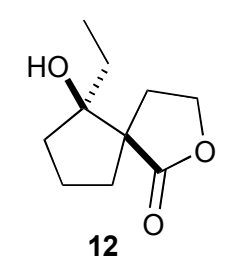

\section{rel-(5R, 6R)-6-Ethyl-6-hydroxy-2-oxa-spiro[4.4]nonan-1-one 12}

As for general procedure A. 3-(E)-(4-Oxo-hexylidene)-dihydro-furan-2-one 7 (40 mg, $0.22 \mathrm{mmol}, 1 \mathrm{eq}$ ), after a reaction time of $1 \mathrm{~h}$ and purification of the crude product

\footnotetext{
${ }^{2}$ Molander, G. A.; Etter, J. B.; Zinke, P. W. J. Am. Chem. Soc. 1987, 109, 453.
} 
mixture by column chromatography (eluting with $60 \%$ EtOAc in petroleum ether (40$\left.60^{\circ} \mathrm{C}\right)$ ), gave rel-(5R, 6R)-6-ethyl-6-hydroxy-2-oxa-spiro[4.4]nonan-1-one 12 (24 mg, $0.13 \mathrm{mmol}, 60 \%)$ as a yellow oil: $\square_{\max }($ neat $) / \mathrm{cm}^{-1} 3477 \mathrm{bs}(\mathrm{OH}), 2968 \mathrm{~s}, 1740 \mathrm{~s}(\mathrm{C}=\mathrm{O})$, $1371 \mathrm{~s}, 1190 \mathrm{~s}, 1032 \mathrm{~m}, 1022 \mathrm{~m}$ and $962 \mathrm{~m} ; \square_{\mathrm{H}}\left(400 \mathrm{MHz}, \mathrm{CDCl}_{3}\right) 0.90(3 \mathrm{H}, \mathrm{t}, J \mathrm{~T} .4$, $\left.\mathrm{CH}_{3}\right), 1.43-1.52\left(2 \mathrm{H}, \mathrm{m}, 1 \mathrm{H}\right.$ from $\mathrm{CH}_{2} \mathrm{CH}_{3}, 1 \mathrm{H}$ from $\left.\mathrm{CH}_{2} \mathrm{C}\right), 1.54-1.62(1 \mathrm{H}, \mathrm{m}, 1 \mathrm{H}$ from $\left.\mathrm{CH}_{2} \mathrm{CH}_{3}\right), 1.63-1.69\left(1 \mathrm{H}, \mathrm{m}, 1 \mathrm{H}\right.$ from $\left.\mathrm{CH}_{2} \mathrm{CH}_{2} \mathrm{C}\right), 1.72-1.78(1 \mathrm{H}, \mathrm{m}, 1 \mathrm{H}$ from $\left.\mathrm{CH}_{2} \mathrm{C}(\mathrm{OH})\right), 1.85-1.96\left(3 \mathrm{H}, \mathrm{m}, 1 \mathrm{H}\right.$ from $\mathrm{CH}_{2} \mathrm{CH}_{2} \mathrm{O}, 1 \mathrm{H}$ from $\mathrm{CH}_{2} \mathrm{C}, 1 \mathrm{H}$ from $\left.\mathrm{CH}_{2} \mathrm{CH}_{2} \mathrm{C}\right), 2.20-2.26\left(2 \mathrm{H}, \mathrm{m}, 1 \mathrm{H}\right.$ from $\mathrm{CH}_{2} \mathrm{C}(\mathrm{OH}), 1 \mathrm{H}$ from $\left.\mathrm{CH}_{2} \mathrm{CH}_{2} \mathrm{O}\right), 3.92(1 \mathrm{H}$, $\mathrm{s}, \mathrm{OH}), 4.15\left(1 \mathrm{H}, \mathrm{dt}, J 6.8,9.2,1 \mathrm{H}\right.$ from $\left.\mathrm{CH}_{2} \mathrm{O}\right)$ and $4.28(1 \mathrm{H}, \mathrm{dt}, J 2.7,9.2,1 \mathrm{H}$ from $\left.\mathrm{CH}_{2} \mathrm{O}\right) ; \square_{\mathrm{C}}\left(100 \mathrm{MHz}, \mathrm{CDCl}_{3}\right) 8.7\left(\mathrm{CH}_{3}\right), 20.7\left(\mathrm{CH}_{2} \mathrm{CH}_{2} \mathrm{C}\right), 29.1\left(\mathrm{CH}_{2} \mathrm{CH}_{3}\right), 30.2$ $\left(\mathrm{CH}_{2} \mathrm{CH}_{2} \mathrm{O}\right), 34.7\left(\mathrm{CH}_{2} \mathrm{C}(\mathrm{OH})\right), 36.9\left(\mathrm{CH}_{2} \mathrm{C}\right), 54.0(C), 65.7\left(\mathrm{CH}_{2} \mathrm{O}\right), 85.1(\mathrm{C}(\mathrm{OH}))$ and $182.9(C(\mathrm{O})) ; \mathrm{m} / \mathrm{z}\left(\mathrm{CI}\right.$ mode, isobutane) $185\left((\mathrm{M}+\mathrm{H})^{+} 100 \%\right), 167(80), 155(2)$, 139 (1), 123 (2), 99 (2), 85 (5) and 71 (5); (Found: $(\mathrm{M}+\mathrm{H})^{+}, 185.1178 . \mathrm{C}_{10} \mathrm{H}_{17} \mathrm{O}_{3}$ requires $M, 185.1178)$.

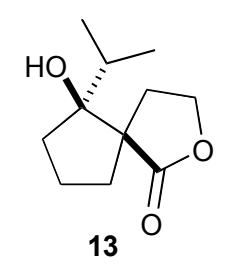

rel-(5R, 6S)-6-Hydroxy-6-isopropyl-2-oxa-spiro[4.4]nonan-1-one 13

As for general procedure A. 3-(E)-(5-Methyl-4-oxo-hexylidene)-dihydro-furan-2-one $\mathbf{8}$ (50 $\mathrm{mg}, 0.26 \mathrm{mmol}, 1 \mathrm{eq})$, after a reaction time of $2 \mathrm{~h}$ and purification of the crude product mixture by column chromatography (eluting with $60 \%$ EtOAc in petroleum ether $\left(40-60^{\circ} \mathrm{C}\right)$ ), gave rel-(5R, 6S)-6-hydroxy-6-isopropyl-2-oxa-spiro[4.4]nonan-1one 13 (24 mg, $0.12 \mathrm{mmol}, 47 \%)$ as a white crystalline solid: $\mathrm{mp} 43-46^{\circ} \mathrm{C}$ (petroleum ether $\left.\left(40-60^{\circ} \mathrm{C}\right)\right): \square_{\max }($ neat $) / \mathrm{cm}^{-1} 3446 \mathrm{bs}(\mathrm{OH}), 2966 \mathrm{~s}, 1734 \mathrm{~s}(\mathrm{C}=\mathrm{O})$, 1470m, 1441m, 1371m, 1186m and 1018m; — $\square_{\mathrm{H}}\left(400 \mathrm{MHz}, \mathrm{CDCl}_{3}\right) 0.75(3 \mathrm{H}, \mathrm{d}, J 6.7$, $\left.\mathrm{CH}\left(\mathrm{CH}_{3}\right)_{2}\right), 0.93\left(3 \mathrm{H}, \mathrm{d}, J\right.$ 6.7, $\left.\mathrm{CH}\left(\mathrm{CH}_{3}\right)_{2}\right), 1.40-1.45\left(1 \mathrm{H}, \mathrm{m}, 1 \mathrm{H}\right.$ from $\left.\mathrm{CH}_{2} \mathrm{C}\right), 1.65$ - $1.70\left(1 \mathrm{H}, \mathrm{m}, 1 \mathrm{H}\right.$ from $\left.\mathrm{CH}_{2} \mathrm{CH}_{2} \mathrm{C}\right), 1.77-1.83\left(3 \mathrm{H}, \mathrm{m}, 1 \mathrm{H}\right.$ from $\mathrm{CH}\left(\mathrm{CH}_{3}\right)_{2}, 1 \mathrm{H}$ from $\left.\mathrm{CH}_{2} \mathrm{C}(\mathrm{OH})\right), 1.91-1.98\left(2 \mathrm{H}, \mathrm{m}, 1 \mathrm{H}\right.$ from $\mathrm{CH}_{2} \mathrm{C}, 1 \mathrm{H}$ from $\left.\mathrm{CH}_{2} \mathrm{CH}_{2} \mathrm{C}\right) 1 \mathrm{H}$ from $\left.\mathrm{CH}_{2} \mathrm{CH}_{2} \mathrm{O}\right), 2.20-2.28\left(2 \mathrm{H}, \mathrm{m}, 1 \mathrm{H}\right.$ from $\left.\mathrm{CH}_{2} \mathrm{C}(\mathrm{OH})\right), 1 \mathrm{H}$ from $\left.\mathrm{CH}_{2} \mathrm{CH}_{2} \mathrm{O}\right), 4.15(1 \mathrm{H}$, 
$\mathrm{dt}, J 5.4,9.1,1 \mathrm{H}$ from $\left.\mathrm{CH}_{2} \mathrm{O}\right), 4.32(1 \mathrm{H}, \mathrm{s}, \mathrm{OH})$ and $4.33(1 \mathrm{H}, \mathrm{dt}, J 1.7,9.1,1 \mathrm{H}$ from $\left.\mathrm{CH}_{2} \mathrm{O}\right)$; $\square_{\mathrm{c}}\left(100 \mathrm{MHz}, \mathrm{CDCl}_{3}\right) 17.4\left(\mathrm{CH}\left(\mathrm{CH}_{3}\right)_{2}\right), 18.4\left(\mathrm{CH}\left(\mathrm{CH}_{3}\right)_{2}\right), 20.8\left(\mathrm{CH}_{2} \mathrm{CH}_{2} \mathrm{C}\right)$, $30.8\left(\mathrm{CH}_{2} \mathrm{CH}_{2} \mathrm{O}\right), 33.7\left(\mathrm{CH}\left(\mathrm{CH}_{3}\right)_{2}\right), 35.5\left(\mathrm{CH}_{2} \mathrm{C}(\mathrm{OH})\right), 38.2\left(\mathrm{CH}_{2} \mathrm{C}\right), 52.8(C), 65.6$ $\left(\mathrm{CH}_{2} \mathrm{O}\right), 87.2(\mathrm{C}(\mathrm{OH}))$ and 183.9( $\left.\mathrm{C}(\mathrm{O})\right) ; \mathrm{m} / z$ (EI mode) $198\left(\left(\mathrm{M}^{+\bullet}\right) 5 \%\right), 180(3), 155$ (100), 152 (5), 127 (10), 99 (45), 83 (80), 71 (15), 55 (20) and 43 (17); (Found: (M+•), 198.1257. $\mathrm{C}_{11} \mathrm{H}_{18} \mathrm{O}_{3}$ requires $\left.M, 198.1256\right)$.

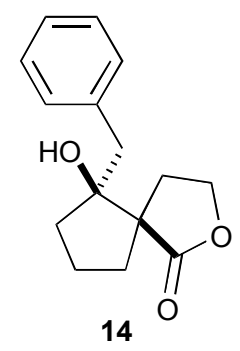

rel-(5R, 6S)-6-Benzyl-6-hydroxy-2-oxa-spiro[4.4]nonan-1-one 14

As for general procedure A. (E)-3-(4-Oxo-5-phenyl-pentylidene)-dihydro-furan-2-one 9 (40 mg, $0.17 \mathrm{mmol}, 1 \mathrm{eq}$ ), after a reaction time of $2 \mathrm{~h}$ and purification of the crude product mixture by column chromatography (eluting with 50\% EtOAc in petroleum ether $\left.\left(40-60^{\circ} \mathrm{C}\right)\right)$, gave rel-(5R, 6S)-6-benzyl-6-hydroxy-2-oxa-spiro[4.4]nonan-1-one $14(27 \mathrm{mg}, 0.12 \mathrm{mmol}, 68 \%)$ as a white crystalline solid: $\mathrm{mp} 70-72^{\circ} \mathrm{C}$ (petroleum ether $\left.\left(40-60^{\circ} \mathrm{C}\right)\right): \square_{\max }($ neat $) / \mathrm{cm}^{-1} 3428 \mathrm{bs}(\mathrm{OH}), 3026 \mathrm{w}, 2978 \mathrm{w}, 1752 \mathrm{~s},(\mathrm{C}=\mathrm{O})$, $1456 \mathrm{~m}, 1380 \mathrm{~m}, 1225 \mathrm{~m}, 1183 \mathrm{~m}$ and $1033 \mathrm{~m}$; $\square_{\mathrm{H}}\left(400 \mathrm{MHz}, \mathrm{CDCl}_{3}\right) 1.61-1.80(4 \mathrm{H}$, m, $\left.\mathrm{CH}_{2} \mathrm{CH}_{2} \mathrm{C}(\mathrm{OH}), \mathrm{CH}_{2} \mathrm{CH}_{2} \mathrm{CH}_{2} \mathrm{C}(\mathrm{OH})\right), 1.89-1.98\left(2 \mathrm{H}, \mathrm{m}, 1 \mathrm{H}\right.$ from $\mathrm{CH}_{2} \mathrm{C}(\mathrm{OH}), 1 \mathrm{H}$ from $\left.\mathrm{CH}_{2} \mathrm{CH}_{2} \mathrm{O}\right), 2.21-2.29\left(1 \mathrm{H}, \mathrm{m}, 1 \mathrm{H}\right.$ from $\left.\mathrm{CH}_{2} \mathrm{C}(\mathrm{OH})\right), 2.46-2.54(1 \mathrm{H}, \mathrm{m}, 1 \mathrm{H}$ from $\left.\mathrm{CH}_{2} \mathrm{CH}_{2} \mathrm{O}\right), 2.78\left(2 \mathrm{H}\right.$, apparent s, $\left.\mathrm{CH}_{2} \mathrm{Ph}\right), 3.78(1 \mathrm{H}, \mathrm{s}, \mathrm{OH}), 4.17(1 \mathrm{H}$, apparent $\mathrm{dt}, J$ 2.6, 9.0, $1 \mathrm{H}$ from $\left.\mathrm{CH}_{2} \mathrm{O}\right), 4.32\left(1 \mathrm{H}\right.$, apparent dt, $J 2.6,9.0,1 \mathrm{H}$ from $\left.\mathrm{CH}_{2} \mathrm{O}\right)$ and 7.14 - $7.24(5 \mathrm{H}, \mathrm{m}, \mathrm{Ar} \mathrm{CH})$; $\square_{\mathrm{c}}\left(100 \mathrm{MHz}, \mathrm{CDCl}_{3}\right) 20.4\left(\mathrm{CH}_{2} \mathrm{C}\right), 31.9\left(\mathrm{CH}_{2} \mathrm{CH}_{2} \mathrm{O}\right)$, $34.6\left(\mathrm{CH}_{2} \mathrm{C}(\mathrm{OH})\right), 36.6\left(\mathrm{CH}_{2} \mathrm{CH}_{2} \mathrm{C}(\mathrm{OH})\right), 41.9\left(\mathrm{CH}_{2} \mathrm{Ph}\right), 54.6(C), 65.6\left(\mathrm{CH}_{2} \mathrm{O}\right), 84.5$ $(C(\mathrm{OH})), 127.1(\mathrm{Ar} C \mathrm{H}), 128.5(2 \times \mathrm{Ar} C \mathrm{H}), 130.7(2 \times \mathrm{Ar} C \mathrm{H}), 137.2(\operatorname{Ar} C)$ and $182.0(C(\mathrm{O})) ; \mathrm{m} / z$ (EI mode) $246\left(\left(\mathrm{M}^{+\bullet}\right) 5 \%\right), 156(15), 155(100), 111(65), 92$ (95), 91 (60), 55 (20) and 41 (15); (Found: $\left(\mathrm{M}^{+\bullet}\right), 246.1257 . \mathrm{C}_{15} \mathrm{H}_{18} \mathrm{O}_{3}$ requires $M$, 246.1256). 


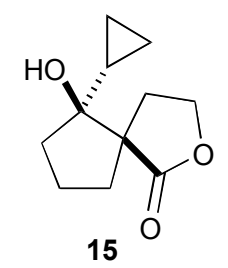

rel-(5R, 6S)-6-Cyclopropyl-6-hydroxy-2-oxa-spiro[4.4]nonan-1-one 15

As for general procedure A. (E)-3-(4-Oxo-4-cyclopropyl-butylidene)-dihydro-furan2-one 10 (30mg, $0.16 \mathrm{mmol}, 1 \mathrm{eq})$, after a reaction time of $2 \mathrm{~h}$ and purification of the crude product mixture by column chromatography (eluting with $20 \%$ EtOAc in petroleum ether $\left.\left(40-60^{\circ} \mathrm{C}\right)\right)$, gave rel-(5R, 6S)-6-cyclopropyl-6-hydroxy-2-oxaspiro[4.4]nonan-1-one 15 (15mg, $0.08 \mathrm{mmol}, 49 \%)$ as a white crystalline solid: $\mathrm{mp} 57$ $-59^{\circ} \mathrm{C}\left(20 \% \mathrm{Et}_{2} \mathrm{O}\right.$ in petroleum ether $\left(30-40^{\circ} \mathrm{C}\right): \square_{\max }($ neat $) / \mathrm{cm}^{-1} 3050(\mathrm{OH}), 2759 \mathrm{w}$, 1743s $(\mathrm{C}=\mathrm{O}), 1384 \mathrm{~m}, 1182 \mathrm{~m}, 1076 \mathrm{~m}, 1018 \mathrm{~m}$ and 953m; $\square_{\mathrm{H}}\left(400 \mathrm{MHz}, \mathrm{CDCl}_{3}\right) 0.27$ $\square 0.37\left(3 \mathrm{H}, \mathrm{m}, 2 \mathrm{H}\right.$ from $\mathrm{C}(\mathrm{OH}) \mathrm{CHCH}_{2}{ }^{\mathrm{A}}, 1 \mathrm{H}$ from $\left.\mathrm{C}(\mathrm{OH}) \mathrm{CHCH}_{2}{ }^{\mathrm{B}}\right), 0.46 \square 0.50(1 \mathrm{H}$, m, $1 \mathrm{H}$ from $\left.\mathrm{C}(\mathrm{OH}) \mathrm{CHCH}_{2}{ }^{\mathrm{B}}\right), 0.80 \square 0.83(1 \mathrm{H}, \mathrm{m}, \mathrm{C}(\mathrm{OH}) \mathrm{CH}), 1.63 \square 1.83(4 \mathrm{H}, \mathrm{m}, 1 \mathrm{H}$ from $\mathrm{CH}_{2} \mathrm{C}(\mathrm{OH}), 3 \mathrm{H}$ from $\left.\mathrm{CH}_{2}\right), 1.89 \square 1.97\left(2 \mathrm{H}, \mathrm{m}, 1 \mathrm{H}\right.$ from $\mathrm{CH}_{2} \mathrm{CH}_{2} \mathrm{O}, 1 \mathrm{H}$ from $\left.\mathrm{CH}_{2}\right), 2.26 \square 2.28\left(1 \mathrm{H}, \mathrm{m}, 1 \mathrm{H}\right.$ from $\left.\mathrm{CH}_{2} \mathrm{C}(\mathrm{OH})\right), 2.50 \square 2.55(1 \mathrm{H}, \mathrm{m}, 1 \mathrm{H}$ from $\left.\mathrm{CH}_{2} \mathrm{CH}_{2} \mathrm{O}\right), 3.62(1 \mathrm{H}, \mathrm{s}, \mathrm{OH}), 4.20\left(1 \mathrm{H}, \mathrm{dt}, J\right.$ 7.2, 8.5, $1 \mathrm{H}$ from $\left.\mathrm{CH}_{2} \mathrm{O}\right)$ and $4.29 \square 4.34$ $\left(1 \mathrm{H}, \mathrm{td}, J\right.$ 4.2, 8.5, $1 \mathrm{H}$ from $\left.\mathrm{CH}_{2} \mathrm{O}\right)$; $\square_{\mathrm{c}}\left(100 \mathrm{MHz}, \mathrm{CDCl}_{3}\right) \square 0.67\left(\mathrm{C}(\mathrm{OH}) \mathrm{CHCH}_{2}{ }^{\mathrm{A}}\right)$, $0.0\left(\mathrm{C}(\mathrm{OH}) \mathrm{CHCH}_{2}{ }^{\mathrm{B}}\right), 14.2(\mathrm{C}(\mathrm{OH}) \mathrm{CH}), 19.9\left(\mathrm{CH}_{2}\right), 31.4\left(\mathrm{CH}_{2} \mathrm{CH}_{2} \mathrm{O}\right), 34.3$ $\left(\mathrm{CH}_{2} \mathrm{C}(\mathrm{OH})\right), 36.6\left(\mathrm{CH}_{2}\right), 54.2(\mathrm{CC}(\mathrm{O})), 65.3\left(\mathrm{CH}_{2} \mathrm{O}\right), 82.2(C(\mathrm{OH}))$ and 182.1 $(C(\mathrm{O})) ; m / z \quad$ (EI mode) $196\left(\left(\mathrm{M}^{+\bullet}\right), 26 \%\right), 112(79), 99(59), 98(32), 85(65), 83$ (100), 69 (50) 48 (19) and 41 (24); (Found: $\left(\mathrm{M}^{+\bullet}\right)$ 196.1100. $\mathrm{C}_{11} \mathrm{H}_{16} \mathrm{O}_{3}$ requires $M$, 196.1099).

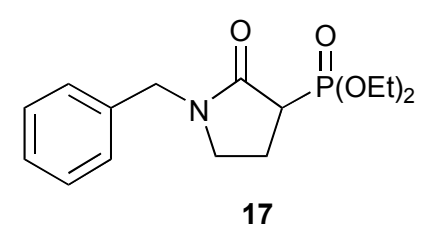




\section{1-Benzyl-3-(diethoxyphosphinyl)-pyrrolidin-2-one 17}

A solution of LDA was prepared by the addition of diisopropylamine (802 $\square 1,5.70$ mmol, 2 eq) to a stirred solution of ${ }^{\mathrm{n}} \mathrm{BuLi}(2.5 \mathrm{M}$ in Hexanes, $2.85 \mathrm{ml}, 5.70 \mathrm{mmol}, 2$ eq) in THF $(2.5 \mathrm{ml})$ at $-5^{\circ} \mathrm{C}$, and the resultant solution was stirred for $30 \mathrm{~min}$. A solution of 1-benzyl-pyrrolidin-2-one (500 mg, $2.85 \mathrm{mmol}, 1 \mathrm{eq})$ in THF (3ml) was then added via cannula, and the temperature raised to $15^{\circ} \mathrm{C}$ and the solution stirred for $10 \mathrm{~min}$. A solution of diethylchlorophosphate (420 $\square 1,2.85 \mathrm{mmol}, 1 \mathrm{eq})$ in THF (2 $\mathrm{ml}$ ) was then added via cannula, whereupon the temperature rose to $25^{\circ} \mathrm{C}$. The resulting solution was stirred at room temperature for $3 \mathrm{~h}$. The solution was then acidified to $\mathrm{pH} 1$ (2 $\mathrm{M}$ aqueous $\mathrm{HCl})$, and the aqueous layer was separated and extracted with $\mathrm{CH}_{2} \mathrm{Cl}_{2}(3 \times 30 \mathrm{ml})$. The combined organic extracts were dried $\left(\mathrm{MgSO}_{4}\right)$, and concentrated in vacuo to give the crude product. Purification by flash column chromatography $\left(50 \%\right.$ EtOAc in petroleum ether $\left(40-60^{\circ} \mathrm{C}\right)$ to remove nonpolar impurities, then with 50\% EtOAc in MeCN) gave 1-benzyl-3(diethoxyphosphinyl)-pyrrolidin-2-one 17 (481 $\mathrm{mg}, 1.55 \mathrm{mmol}, 54 \%)$ as a brown oil: $\square_{\max }($ neat $) / \mathrm{cm}^{-1} 2983 \mathrm{~s}, 2909 \mathrm{~s}, 1693 \mathrm{~s}(\mathrm{C}=\mathrm{O}), 1496 \mathrm{~m}, 1455 \mathrm{~m}, 1440 \mathrm{~m}, 1258 \mathrm{~m}(\mathrm{P}=\mathrm{O})$, $1164 \mathrm{w}, 1059 \mathrm{~m}(\mathrm{P}-\mathrm{O})$ and $961 \mathrm{~m} ; \square_{\mathrm{H}}\left(400 \mathrm{MHz}, \mathrm{CDCl}_{3}\right) 1.26\left(3 \mathrm{H}, \mathrm{t}, J 7.1, \mathrm{OCH}_{2} \mathrm{CH}_{3}\right)$, $1.29\left(3 \mathrm{H}, \mathrm{t}, J\right.$ 7.1, $\left.\mathrm{OCH}_{2} \mathrm{CH}_{3}\right), 2.19-2.37\left(2 \mathrm{H}, \mathrm{m}, \mathrm{CH}_{2} \mathrm{CHP}\right), 2.94\left(1 \mathrm{H}, \mathrm{ddd}, J_{\mathrm{HP}}, 22.0\right.$, $J$ 5.3, 10.2, CHP), $3.12-3.18\left(1 \mathrm{H}, \mathrm{m}, 1 \mathrm{H}\right.$ from $\left.\mathrm{CH}_{2} \mathrm{~N}\right), 3.33(1 \mathrm{H}$, apparent q, $J$ 7.7, $1 \mathrm{H}$ from $\left.\mathrm{CH}_{2} \mathrm{~N}\right), 4.08-4.23\left(4 \mathrm{H}, \mathrm{m}, 2 \times \mathrm{OCH}_{2}\right), 4.36(1 \mathrm{H}, \mathrm{d}, J 14.8, \mathrm{AB}$ system, $1 \mathrm{H}$ from $\left.\mathrm{CH}_{2} \mathrm{Ph}\right), 4.46\left(1 \mathrm{H}, \mathrm{d}, J\right.$ 14.8, AB system, $1 \mathrm{H}$ from $\left.\mathrm{CH}_{2} \mathrm{Ph}\right), 7.15-7.23(3 \mathrm{H}, \mathrm{m}, 3$ $\mathrm{x} \mathrm{Ar} \mathrm{CH})$ and $7.24-7.28(2 \mathrm{H}, \mathrm{m}, 2 \mathrm{x} \mathrm{Ar} \mathrm{CH})$; $\square_{\mathrm{C}}\left(100 \mathrm{MHz}, \mathrm{CDCl}_{3}\right) 16.8\left(\mathrm{~d}, J_{\mathrm{CP}} 4.1\right.$, $\left.\mathrm{OCH}_{2} \mathrm{CH}_{3}\right), 16.8\left(\mathrm{~d}, J_{\mathrm{CP}} 4.1, \mathrm{OCH}_{2} \mathrm{CH}_{3}\right), 20.6\left(\mathrm{~d}, J_{\mathrm{CP}} 3.9, \mathrm{NCH}_{2} \mathrm{CH}_{2}\right), 41.4\left(\mathrm{~d}, J_{\mathrm{CP}}\right.$ 141.2, CHP), $45.7\left(\mathrm{~d}, J_{\mathrm{CP}} 3.9, \mathrm{NCH}_{2}\right), 47.4\left(\mathrm{CH}_{2} \mathrm{Ph}\right), 62.7\left(\mathrm{~d}, J_{\mathrm{CP}} 6.6, \mathrm{OCH}_{2}\right), 63.4(\mathrm{~d}$, $J_{\mathrm{CP}}$ 6.6, $\left.\mathrm{OCH}_{2}\right), 128.0(\mathrm{Ar} C \mathrm{H}), 128.5(2 \times \mathrm{Ar} C \mathrm{H}), 129.1(2 \times \mathrm{Ar} C \mathrm{H}), 136.4(\mathrm{Ar} C)$ and $169.7\left(\mathrm{~d}, J_{\mathrm{CP}} 4.0 \mathrm{C}(\mathrm{O})\right)$; 口 $\left(81 \mathrm{MHz}, \mathrm{CDCl}_{3}\right)$ 24.4; $\mathrm{m} / \mathrm{z}(\mathrm{EI}$ mode $) 311\left(\left(\mathrm{M}^{+\bullet}\right)\right.$ 80\%), 282 (10), 254 (5), 238 (2), 220 (15), 179 (15), 174 (40), 145 (20), 119 (25), 91 (100), 83 (30), 65 (10) and 47 (7); (Found: $\left(\mathrm{M}^{+\bullet}\right), 311.1285 . \mathrm{C}_{15} \mathrm{H}_{22} \mathrm{O}_{4} \mathrm{NP}$ requires $M$, 311.1286). 


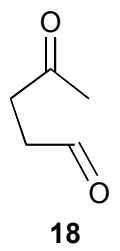

\section{4-Oxo-pentanal $18^{3}$}

To a stirred solution of 3-acetylpropanol (5.00 g, $50.0 \mathrm{mmol}, 1 \mathrm{eq})$ in $\mathrm{CH}_{2} \mathrm{Cl}_{2}(150 \mathrm{ml})$ at room temperature was added pyridinium chlorochromate $(16.2 \mathrm{~g}, 75.0 \mathrm{mmol}, 1.5$ eq) and the resulting solution stirred for $16 \mathrm{~h}$. The reaction mixture was passed through a plug of silica and concentrated in vacuo to give the crude product. Purification by column chromatography (eluting with $\mathrm{CH}_{2} \mathrm{Cl}_{2}$ ) gave 4-oxo-pentanal 18 (3.53 g, $35.3 \mathrm{mmol}, 71 \%$ ) as a clear, colourless oil: $\square_{\max }($ neat $) / \mathrm{cm}^{-1} 2729 \mathrm{~s}, 1716 \mathrm{bs}(2$ x C=O), 1428bs, 1170w, 1132w, 1080w, 961m and 879m; $\square_{\mathrm{H}}\left(400 \mathrm{MHz}, \mathrm{CDCl}_{3}\right) 2.23$ $\left(3 \mathrm{H}, \mathrm{s}, \mathrm{CH}_{3}\right), 2.77\left(4 \mathrm{H}, \mathrm{s}, 2 \times \mathrm{CH}_{2}\right)$ and $9.83(1 \mathrm{H}, \mathrm{s}, \mathrm{CHO})$; $\square_{\mathrm{c}}\left(100 \mathrm{MHz}, \mathrm{CDCl}_{3}\right) 30.2$ $\left(\mathrm{CH}_{3}\right), 35.9\left(\mathrm{CH}_{2} \mathrm{C}(\mathrm{O}) \mathrm{CH}_{3}\right), 27.8\left(\mathrm{CH}_{2} \mathrm{CHO}\right), 200.8(\mathrm{CHO})$ and $206.8(\mathrm{C}(\mathrm{O})) ; \mathrm{m} / \mathrm{z}(\mathrm{CI}$ mode, isobutane) $101\left((\mathrm{M}+\mathrm{H})^{+} 20 \%\right), 57(100)$ and $56(10)$; (Found: $(\mathrm{M}+\mathrm{H})^{+}$, 101.0602. $\mathrm{C}_{5} \mathrm{H}_{9} \mathrm{O}_{2}$ requires $\left.M, 101.0603\right)$.

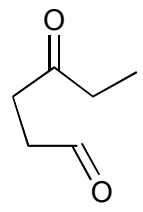

19

\section{4-Oxo-hexanal $19^{4}$}

DMSO (361 $\square 1,5.08 \mathrm{mmol}, 4 \mathrm{eq})$ was added to a stirred solution of $(\mathrm{COCl})_{2}(222 \square 1$, $2.54 \mathrm{mmol}, 2 \mathrm{eq})$ in $\mathrm{CH}_{2} \mathrm{Cl}_{2}(10 \mathrm{ml})$ at $-78^{\circ} \mathrm{C}$ and the resulting solution was stirred for $10 \mathrm{~min}$. Hexane-1,4-diol (150 mg, $1.27 \mathrm{mmol}, 1 \mathrm{eq})$ was then added as a solution in $\mathrm{CH}_{2} \mathrm{Cl}_{2}(5 \mathrm{ml})$ and the resultant solution stirred for a further $30 \mathrm{~min}$. Triethylamine $(1.82 \mathrm{ml}, 12.7 \mathrm{mmol}, 10 \mathrm{eq})$ was then added and the reaction mixture was allowed to warm to room temperature and stir for $2 \mathrm{~h}$. Aqueous saturated $\mathrm{NaHCO}_{3}(5 \mathrm{ml})$ was

\footnotetext{
${ }^{3}$ Molander, G. A.; Cameron, K. O. J. Am. Chem. Soc. 1993, 115, 830.

${ }^{4}$ Takeoka, G. R.; Buttery, R. G.; Perrino, C. T. J. Agric. Food Chem. 1995, 43, 22.
} 
then added, and the aqueous layer was separated and extracted with EtOAc (4 x 10 $\mathrm{ml})$. The combined organic extracts were dried $\left(\mathrm{MgSO}_{4}\right)$, and concentrated in vacuo to give the crude product. Purification by column chromatography (eluting with $80 \%$ EtOAc in petroleum ether $\left.\left(40-60^{\circ} \mathrm{C}\right)\right)$ gave 4-oxo-hexanal $19(103 \mathrm{mg}, 0.90 \mathrm{mmol}$, $73 \%$ ) as a pale yellow oil: $\square_{\max }$ (neat) $/ \mathrm{cm}^{-1} 2980 \mathrm{~s}, 1735$ s (aldehyde $\mathrm{C}=\mathrm{O}$ ), 1719s (ketone $\mathrm{C}=\mathrm{O}), 1459 \mathrm{~s}, 1420 \mathrm{~s}, 1116 \mathrm{~m}$ and $1089 \mathrm{~m} ; \square_{\mathrm{H}}\left(400 \mathrm{MHz}, \mathrm{CDCl}_{3}\right) 1.01(3 \mathrm{H}, \mathrm{t}, J$ 7.3, $\left.\mathrm{C}(\mathrm{O}) \mathrm{CH}_{2} \mathrm{CH}_{3}\right), 2.41\left(2 \mathrm{H}, \mathrm{q}, J 7.3, \mathrm{C}(\mathrm{O}) \mathrm{CH}_{2}\right), 2.64-2.72\left(4 \mathrm{H}, \mathrm{m}, 2 \times \mathrm{CH}_{2}\right)$ and $9.70(1 \mathrm{H}, \mathrm{s}, \mathrm{CHO})$; $\square_{\mathrm{C}}\left(100 \mathrm{MHz}, \mathrm{CDCl}_{3}\right) 8.2\left(\mathrm{CH}_{3}\right), 34.6\left(\mathrm{CH}_{2}\right), 36.3\left(\mathrm{C}(\mathrm{O}) \mathrm{CH}_{2}\right)$, $37.9\left(\mathrm{CH}_{2}\right), 200.9(\mathrm{CHO})$ and $209.6(\mathrm{C}(\mathrm{O})) ; \mathrm{m} / z$ (CI mode, isobutane) $127(10 \%), 115$ $\left((\mathrm{M}+\mathrm{H})^{+} 100\right), 99$ (95), 97 (5) and 71 (5); (Found: $(\mathrm{M}+\mathrm{H})^{+}, 115.0759 . \mathrm{C}_{6} \mathrm{H}_{11} \mathrm{O}_{2}$ requires $M, 115.0759)$.

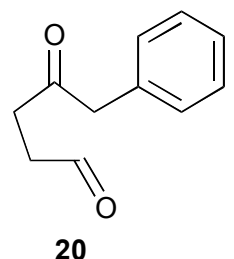

\section{4-Oxo-5-phenyl-pentanal $20^{5}$}

DMSO (261 $\square 1,3.68 \mathrm{mmol}, 4 \mathrm{eq})$ was added to a stirred solution of $(\mathrm{COCl})_{2}(161 \square 1$, $1.84 \mathrm{mmol}, 2$ eq) in $\mathrm{CH}_{2} \mathrm{Cl}_{2}(7 \mathrm{ml})$ at $-78^{\circ} \mathrm{C}$ and the resulting solution was stirred for $10 \mathrm{~min}$. 5-Phenyl-pentane-1,4-diol (165 mg, $0.92 \mathrm{mmol}, 1 \mathrm{eq})$ was then added as a solution in $\mathrm{CH}_{2} \mathrm{Cl}_{2}(3 \mathrm{ml})$ and the resultant solution stirred for a further $30 \mathrm{~min}$. Triethylamine (1.32 ml, $9.2 \mathrm{mmol}, 10 \mathrm{eq})$ was then added and the reaction mixture was allowed to warm to room temperature and stir for $2 \mathrm{~h}$. Aqueous saturated $\mathrm{NaHCO}_{3}(5 \mathrm{ml})$ was then added, and the aqueous layer was separated and extracted with EtOAc $(4 \times 10 \mathrm{ml})$. The combined organic extracts were dried $\left(\mathrm{MgSO}_{4}\right)$, and concentrated in vacuo to give the crude product. Purification by column chromatography (eluting with $20 \%$ EtOAc in petroleum ether $\left(40-60^{\circ} \mathrm{C}\right)$ ) gave 4-oxo5-phenyl-pentanal 20 (116 mg, $0.66 \mathrm{mmol}, 72 \%)$ as a pale yellow oil: $\square_{\max }($ neat $) / \mathrm{cm}^{-1}$ 2826m, 2727m, 1702bs ( 2 x C=O), 1603m, 1584m, 1498m, 1454m, 1413m, 1092m, $1036 \mathrm{~m}$ and $1002 \mathrm{w} ; \square_{\mathrm{H}}\left(400 \mathrm{MHz}, \mathrm{CDCl}_{3}\right) 2.64-2.72\left(4 \mathrm{H}, \mathrm{m}, 2 \times \mathrm{CH}_{2}\right), 3.69(2 \mathrm{H}, \mathrm{s}$,

\footnotetext{
${ }^{5}$ Brown, E.; Paterne, M. Bull. Soc. Chim. Fr. 1974, 1001.
} 
$\left.\mathrm{CH}_{2} \mathrm{Ph}\right), 7.14-7.35(5 \mathrm{H}, \mathrm{m}, 5 \mathrm{x} \mathrm{Ar} \mathrm{CH})$ and $9.71(1 \mathrm{H}, \mathrm{s}, \mathrm{CHO}) ; \square_{\mathrm{c}}(100 \mathrm{MHz}$, $\left.\mathrm{CDCl}_{3}\right) 34.4\left(\mathrm{CH}_{2}\right), 37.9\left(\mathrm{CH}_{2}\right), 50.4\left(\mathrm{CH}_{2} \mathrm{Ph}\right), 127.5(\mathrm{Ar} \mathrm{CH}), 129.2(2 \times \mathrm{Ar} \mathrm{CH})$, $129.8(2 \times \mathrm{Ar} C \mathrm{H}), 134.4(\mathrm{Ar} C), 200.7(\mathrm{CHO})$ and $206.6(\mathrm{C}(\mathrm{O})) ; \mathrm{m} / z$ (EI mode) 176 ((M+•) 20\%), 165 (5), 148 (5), 117 (3), 115 (3), 85 (100), 65 (15), 57 (10) and 51 (5); (Found: $\left(\mathrm{M}^{+\bullet}\right), 176.0836 . \mathrm{C}_{11} \mathrm{H}_{12} \mathrm{O}_{2}$ requires $M, 176.0837$ ).

\section{General Horner-Wittig Olefination Procedure C}

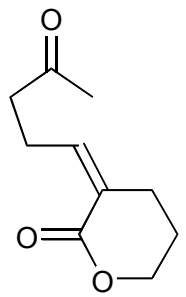

$21 Z$

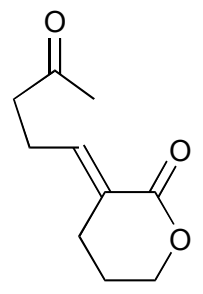

$21 E$

(Z)-3-(4-Oxo-pentylidene)-tetrahydro-pyran-2-one $21 Z$ and (E)-3-(4-Oxopentylidene)-tetrahydro-pyran-2-one $21 E$

$\mathrm{K}_{2} \mathrm{CO}_{3}(643 \mathrm{mg}, 4.65 \mathrm{mmol}, 1.1 \mathrm{eq})$ was added to a solution of 18-crown-6 (1.23 g, $4.65 \mathrm{mmol}, 1.1 \mathrm{eq})$ and 3-(diethoxyphosphinyl)-tetrahydro-pyran-2-one 16 (1.00 g, $4.23 \mathrm{mmol}, 1 \mathrm{eq})$ in THF $(50 \mathrm{ml})$ at room temperature, and the resultant solution stirred for 2 h. 4-Oxo-pentanal 18 (509 mg, $5.08 \mathrm{mmol}, 1.2 \mathrm{eq})$ in THF (10 ml) was then added and the solution stirred for $16 \mathrm{~h}$. Aqueous saturated $\mathrm{NH}_{4} \mathrm{Cl}(30 \mathrm{ml})$ was then added dropwise, and the aqueous layer was separated and extracted with $\mathrm{Et}_{2} \mathrm{O}$ (3 x $50 \mathrm{ml})$. The combined organic extracts were dried $\left(\mathrm{MgSO}_{4}\right)$, and concentrated in vacuo to give the crude product. Purification by flash column chromatography (eluting with EtOAc) gave (Z)-3-(4-oxo-pentylidene)-tetrahydro-pyran-2-one $\mathbf{2 1 Z}$ (301 mg, $1.65 \mathrm{mmol}, 39 \%$ ) as a pale yellow oil: $\square_{\max }$ (neat) $/ \mathrm{cm}^{-1} 2928 \mathrm{~s}, 2861 \mathrm{~s}, 1733 \mathrm{~s}$ $(\mathrm{C}=\mathrm{O}), 1635 \mathrm{~s}(\mathrm{C}=\mathrm{C}), 1478 \mathrm{~m}, 1449 \mathrm{~m}, 1116 \mathrm{~m}, 1061 \mathrm{~m}, 1028 \mathrm{~m}$ and $976 \mathrm{~m} ; \square_{\mathrm{H}}(400$ $\left.\mathrm{MHz}, \mathrm{CDCl}_{3}\right) 1.72-1.76\left(2 \mathrm{H}, \mathrm{m}, \mathrm{OCH}_{2} \mathrm{CH}_{2}\right), 1.99\left(3 \mathrm{H}, \mathrm{s}, \mathrm{C}(\mathrm{O}) \mathrm{CH}_{3}\right), 2.39(2 \mathrm{H}, \mathrm{dt}, J$ 1.6, 5.2, $\left.\mathrm{CH}=\mathrm{CCH}_{2}\right), 2.46\left(2 \mathrm{H}, \mathrm{t}, J\right.$ 7.0, $\left.\mathrm{CH}_{2} \mathrm{C}(\mathrm{O})\right), 2.65(2 \mathrm{H}$, apparent q, $J$ 7.0, $\left.\mathrm{CH}_{2} \mathrm{CH}=\mathrm{C}\right), 4.11\left(2 \mathrm{H}, \mathrm{t}, \mathrm{J} 5.4, \mathrm{OCH}_{2}\right)$ and $5.93(1 \mathrm{H}, \mathrm{tt}, J 1.6,7.5, \mathrm{CH}=\mathrm{C}) ; \square_{\mathrm{C}}(100$ 
$\left.\mathrm{MHz}, \mathrm{CDCl}_{3}\right) 23.7\left(\mathrm{OCH}_{2} \mathrm{CH}_{2}\right), 24.5\left(\mathrm{CH}_{2} \mathrm{CH}=\mathrm{C}\right), 29.7\left(\mathrm{CH}=\mathrm{CCH}_{2}\right), 30.1\left(\mathrm{C}(\mathrm{O}) \mathrm{CH}_{3}\right)$, $43.2\left(\mathrm{CH}_{2} \mathrm{C}(\mathrm{O})\right), 69.1\left(\mathrm{OCH}_{2}\right), 126.2(\mathrm{CH}=C), 146.1(\mathrm{CH}=\mathrm{C}), 165.9(C(\mathrm{O}) \mathrm{O})$ and $208.5(C(\mathrm{O}))$.

Further elution gave (E)-3-(4-oxo-pentylidene)-tetrahydro-pyran-2-one $\mathbf{2 1 E}$ (120 mg, $0.66 \mathrm{mmol}, 16 \%)$ as a pale yellow oil: $\square_{\max }($ neat $) / \mathrm{cm}^{-1} 2901 \mathrm{~s}, 1727 \mathrm{~s}(\mathrm{C}=\mathrm{O}), 1636 \mathrm{~s}$ $(\mathrm{C}=\mathrm{C}), 1479 \mathrm{~m}, 1438 \mathrm{~m}, 1313 \mathrm{~m}, 1274 \mathrm{~m}, 1159 \mathrm{~m}, 1129 \mathrm{~m}, 1073 \mathrm{~m}$ and $967 \mathrm{~m}$; $\mathrm{L}_{\mathrm{H}}(400$ $\left.\mathrm{MHz}, \mathrm{CDCl}_{3}\right) 1.75-1.81\left(2 \mathrm{H}, \mathrm{m}, \mathrm{CH}_{2} \mathrm{CH}_{2} \mathrm{O}\right), 2.01\left(3 \mathrm{H}, \mathrm{s}, \mathrm{C}(\mathrm{O}) \mathrm{CH}_{3}\right), 2.24(2 \mathrm{H}$, apparent q, $J$ 7.2, $\left.\mathrm{CH}_{2} \mathrm{CH}=\mathrm{C}\right), 2.42\left(2 \mathrm{H}, \mathrm{dt}, J\right.$ 2.1, 7.2, $\left.\mathrm{CH}=\mathrm{CCH}_{2}\right), 2.47(2 \mathrm{H}, \mathrm{t}, J$ 7.2, $\left.\mathrm{CH}_{2} \mathrm{C}(\mathrm{O})\right), 4.15\left(2 \mathrm{H}, \mathrm{t}, J 5.3, \mathrm{CH}_{2} \mathrm{O}\right)$ and $6.75(1 \mathrm{H}, \mathrm{tt}, J$ 2.1, 7.3, $\mathrm{CH}=\mathrm{C}) ; \square_{\mathrm{C}}(100$ $\left.\mathrm{MHz}, \mathrm{CDCl}_{3}\right) 22.6\left(\mathrm{CH}_{2} \mathrm{CH}=\mathrm{C}\right), 23.0\left(\mathrm{CH}_{2} \mathrm{CH}_{2} \mathrm{O}\right), 24.0\left(\mathrm{CH}=\mathrm{CCH}_{2}\right), 30.4\left(\mathrm{C}(\mathrm{O}) \mathrm{CH}_{3}\right)$, $42.0\left(\mathrm{CH}_{2} \mathrm{C}(\mathrm{O})\right), 69.0\left(\mathrm{CH}_{2} \mathrm{O}\right), 126.9(\mathrm{CH}=C), 144.5(\mathrm{CH}=\mathrm{C}), 166.8(C(\mathrm{O}) \mathrm{O})$ and $207.3(C(\mathrm{O})) ; \mathrm{m} / \mathrm{z}\left(\mathrm{EI}\right.$ mode) $182\left(\left(\mathrm{M}^{+\bullet}\right)\right.$ 15\%), $164(1), 139$ (100), 125 (5), $111(10)$, 93 (15), 84 (15), 67 (15) and 43 (35); (Found: $\left(\mathrm{M}^{+\bullet}\right)$, 182.0942. $\mathrm{C}_{10} \mathrm{H}_{14} \mathrm{O}_{3}$ requires $M$, 182.0943).

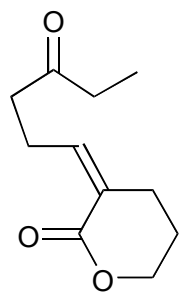

$22 Z$

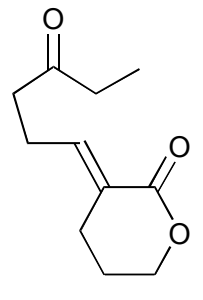

$22 E$

(Z)-3-(4-Oxo-hexylidene)-tetrahydro-pyran-2-one $22 Z$ and (E)-3-(4-Oxohexylidene)-tetrahydro-pyran-2-one $22 E$

As for general procedure C. Reaction of 3-(diethoxyphosphinyl)-tetrahydro-pyran-2one 16 (207 mg, $0.88 \mathrm{mmol}, 1 \mathrm{eq}$ ) with 4-oxo-hexanal 19 (100 mg, $0.88 \mathrm{mmol}, 1 \mathrm{eq})$ for $18 \mathrm{~h}$, after purification by column chromatography (eluting with $80 \%$ EtOAc in petroleum ether $\left(40-60^{\circ} \mathrm{C}\right)$ ), gave $(Z)-3$-(4-Oxo-hexylidene)-tetrahydro-pyran-2-one $22 Z$ (29 mg, $0.15 \mathrm{mmol}, 17 \%)$ as a yellow oil: $\square_{\max }($ neat $) / \mathrm{cm}^{-1} 2974 \mathrm{w}, 1709 \mathrm{~s}(\mathrm{C}=\mathrm{O})$, $1633 \mathrm{~s}(\mathrm{C}=\mathrm{C}), 1398 \mathrm{~m}, 1375 \mathrm{~m}, 1124 \mathrm{~m}$ and $1072 \mathrm{~m}$; $\square_{\mathrm{H}}\left(400 \mathrm{MHz}, \mathrm{CDCl}_{3}\right) 0.99(3 \mathrm{H}, \mathrm{t}, J$ 7.3, $\left.\mathrm{CH}_{2} \mathrm{CH}_{3}\right), 1.85\left(2 \mathrm{H}\right.$, apparent quintet, $\left.J 6.6, \mathrm{OCH}_{2} \mathrm{CH}_{2}\right), 2.36(2 \mathrm{H}, \mathrm{q}, J$ 7.3, $\left.\mathrm{CH}_{2} \mathrm{CH}_{3}\right), 2.48,\left(2 \mathrm{H}, \mathrm{dt}, J 1.6,7.1, \mathrm{CH}=\mathrm{CCH}_{2}\right), 2.53\left(2 \mathrm{H}, \mathrm{t}, J 7.1 \mathrm{CH}_{2} \mathrm{C}(\mathrm{O})\right), 2.76(2 \mathrm{H}$, 
apparent q, $J$ 7.1, $\left.\mathrm{CH}_{2} \mathrm{CH}=\mathrm{C}\right), 4.21\left(2 \mathrm{H}, \mathrm{t}, J 5.5, \mathrm{CH}_{2} \mathrm{O}\right)$ and $6.03(1 \mathrm{H}, \mathrm{tt}, J$ 1.6, 7.6, $\mathrm{CH}=\mathrm{C}) ; \mathrm{Z}_{\mathrm{c}}\left(100 \mathrm{MHz}, \mathrm{CDCl}_{3}\right) 8.2\left(\mathrm{CH}_{3}\right), 23.7\left(\mathrm{OCH}_{2} \mathrm{CH}_{2}\right), 24.6\left(\mathrm{CH}_{2} \mathrm{CH}=\mathrm{C}\right), 29.7$ $\left(\mathrm{CH}=\mathrm{CCH}_{2}\right), 30.1\left(\mathrm{CH}_{2} \mathrm{CH}_{3}\right), 41.8\left(\mathrm{CH}_{2} \mathrm{C}(\mathrm{O})\right), 69.1\left(\mathrm{CH}_{2} \mathrm{O}\right), 126.1(\mathrm{CH}=\mathrm{C}), 146.4$ $(C \mathrm{H}=\mathrm{C}), 166.0(C(\mathrm{O}) \mathrm{O})$ and $211.2(C(\mathrm{O}))$.

Further elution gave (E)-3-(4-Oxo-hexylidene)-tetrahydro-pyran-2-one $\mathbf{2 2 E}$ (76 mg, $0.39 \mathrm{mmol}, 44 \%)$ as a yellow oil: $\square_{\max }($ neat $) / \mathrm{cm}^{-1} 2974 \mathrm{~m}, 1704 \mathrm{~s}(\mathrm{C}=\mathrm{O}), 1632 \mathrm{~s}(\mathrm{C}=\mathrm{C})$, $1394 \mathrm{~m}, 1254 \mathrm{~m}, 1169 \mathrm{~m}$ and $1074 \mathrm{~m} ; \square_{\mathrm{H}}\left(400 \mathrm{MHz}, \mathrm{CDCl}_{3}\right) 1.00(3 \mathrm{H}, \mathrm{t}, J \mathrm{~J} .3$, $\left.\mathrm{CH}_{2} \mathrm{CH}_{3}\right), 1.86\left(2 \mathrm{H}\right.$, apparent quintet, $J$ 6.6, $\left.\mathrm{CH}_{2} \mathrm{CH}_{2} \mathrm{O}\right), 2.33(2 \mathrm{H}$, apparent $\mathrm{t}, J$ 7.4, $\left.\mathrm{CH}_{2} \mathrm{CH}=\mathrm{C}\right), 2.38\left(2 \mathrm{H}, \mathrm{q}, J\right.$ 7.3, $\left.\mathrm{CH}_{2} \mathrm{CH}_{3}\right), 2.52\left(2 \mathrm{H}, \mathrm{dt}, J 2.3,7.4, \mathrm{CH}=\mathrm{CCH}_{2}\right), 2.54$ $\left(2 \mathrm{H}, \mathrm{t}, J\right.$ 7.4, $\left.\mathrm{CH}_{2} \mathrm{C}(\mathrm{O})\right), 4.26\left(2 \mathrm{H}, \mathrm{t}, J 5.8, \mathrm{CH}_{2} \mathrm{O}\right)$ and $6.84(1 \mathrm{H}, \mathrm{tt}, J 2.3,7.4, \mathrm{CH}=\mathrm{C})$; $\mathrm{Z}_{\mathrm{c}}\left(100 \mathrm{MHz}, \mathrm{CDCl}_{3}\right) 8.1\left(\mathrm{CH}_{2} \mathrm{CH}_{3}\right), 22.7\left(\mathrm{CH}_{2} \mathrm{CH}=\mathrm{C}\right), 23.0\left(\mathrm{CH}_{2} \mathrm{CH}_{2} \mathrm{O}\right), 24.0$ $\left(\mathrm{CH}=\mathrm{CCH}_{2}\right), 36.4\left(\mathrm{CH}_{2} \mathrm{CH}_{3}\right), 40.6\left(\mathrm{CH}_{2} \mathrm{C}(\mathrm{O})\right), 69.0\left(\mathrm{CH}_{2} \mathrm{O}\right), 126.8(\mathrm{CH}=C), 144.8$ $(C \mathrm{H}=\mathrm{C}), 166.9(C(\mathrm{O}) \mathrm{O})$ and $210.1(C(\mathrm{O})) ; \mathrm{m} / \mathrm{z}(\mathrm{EI}$ mode $) 196\left(\left(\mathrm{M}^{+\bullet}\right) 55 \%\right), 167(10)$, 139 (100), 125 (20), 111 (30), 93 (50), 67 (40), 57 (65) and 41 (25); (Found: (M+•), 196.1100. $\mathrm{C}_{11} \mathrm{H}_{16} \mathrm{O}_{3}$ requires $\left.M, 196.1099\right)$.

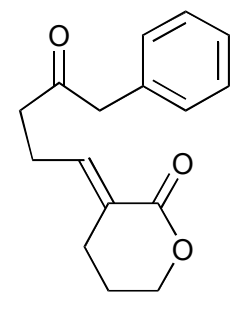

$23 E$

\section{(E)-3-(4-Oxo-5-phenyl-pentylidene)-tetrahydro-pyran-2-one 23E}

As for general procedure C. Reaction of 3-(diethoxyphosphinyl)-tetrahydro-pyran-2one 16 (135 mg, $0.57 \mathrm{mmol}, 1 \mathrm{eq}$ ) with 4-oxo-5-phenyl-pentanal 20 (100 mg, 0.57 mmol, $1 \mathrm{eq}$ ) for $18 \mathrm{~h}$, after purification by column chromatography (eluting with $80 \%$ EtOAc in petroleum ether $\left(40-60^{\circ} \mathrm{C}\right)$, gave $(E)-3-(4-O x o-5-$ phenyl-pentylidene)tetrahydro-pyran-2-one $\mathbf{2 3 E}$ (68 $\mathrm{mg}, 0.26 \mathrm{mmol}, 46 \%$ ) as a yellow oil: $\square_{\max }$ (neat)/ $\mathrm{cm}^{-1}$ $2897 \mathrm{w}, 1707 \mathrm{~s}(\mathrm{C}=\mathrm{O}), 1632 \mathrm{~s}(\mathrm{C}=\mathrm{C}), 1496 \mathrm{~m}, 1396 \mathrm{~m}, 1259 \mathrm{~m}, 1167 \mathrm{~m}$ and 1074m; 口 (400 $\left.\mathrm{MHz}, \mathrm{CDCl}_{3}\right) 1.84\left(2 \mathrm{H}\right.$, apparent quintet, $J$ 6.5, $\left.\mathrm{CH}_{2} \mathrm{CH}_{2} \mathrm{O}\right), 2.29(2 \mathrm{H}$, apparent $\mathrm{q}, J$ 7.2, $\left.\mathrm{CH}_{2} \mathrm{CH}=\mathrm{C}\right), 2.46\left(2 \mathrm{H}\right.$, apparent $\left.\mathrm{t}, J 7.2, \mathrm{CH}=\mathrm{CCH}_{2}\right), 2.56(2 \mathrm{H}, \mathrm{t}, J 7.2$, 
$\left.\mathrm{CH}_{2} \mathrm{C}(\mathrm{O})\right), 3.62\left(2 \mathrm{H}, \mathrm{s}, \mathrm{CH}_{2} \mathrm{Ph}\right), 4.22\left(2 \mathrm{H}, \mathrm{t}, J\right.$ 5.4, $\left.\mathrm{CH}_{2} \mathrm{O}\right), 6.78(1 \mathrm{H}, \mathrm{tt}, J$ 2.4, 7.2, $\mathrm{CH}=\mathrm{C})$ and $7.05-7.32(5 \mathrm{H}, \mathrm{m}, \mathrm{Ar} \mathrm{CH})$; $\mathrm{Z}_{\mathrm{c}}\left(100 \mathrm{MHz}, \mathrm{CDCl}_{3}\right) 22.7\left(\mathrm{CH}_{2} \mathrm{CH}=\mathrm{C}\right)$, $23.0\left(\mathrm{CH}_{2} \mathrm{CH}_{2} \mathrm{O}\right), 24.0\left(\mathrm{CH}=\mathrm{CCH}_{2}\right), 40.3\left(\mathrm{CH}_{2} \mathrm{C}(\mathrm{O})\right), 50.7\left(\mathrm{CH}_{2} \mathrm{Ph}\right), 69.0\left(\mathrm{CH}_{2} \mathrm{O}\right)$, 126.9 $(\mathrm{CH}=C), 127.6(\operatorname{Ar} C H), 129.2(2 \times \operatorname{Ar} C H), 129.8(2 \times \operatorname{Ar} C H), 134.3(\operatorname{Ar} C)$, $144.4(\mathrm{CH}=\mathrm{C}), 166.8(C(\mathrm{O}) \mathrm{O})$ and $207.1(C(\mathrm{O})) ; \mathrm{m} / z(\mathrm{EI}$ mode $) 258\left(\left(\mathrm{M}^{+\bullet}\right) 35 \%\right), 184$ (2), 167 (100), 139 (30), 121 (15), 91 (70), 65 (15) and 55 (10); (Found: $\left(\mathrm{M}^{\mathbf{+}^{\bullet}}\right.$ ), 258.1256. $\mathrm{C}_{16} \mathrm{H}_{18} \mathrm{O}_{3}$ requires $\left.M, 258.1256\right)$.
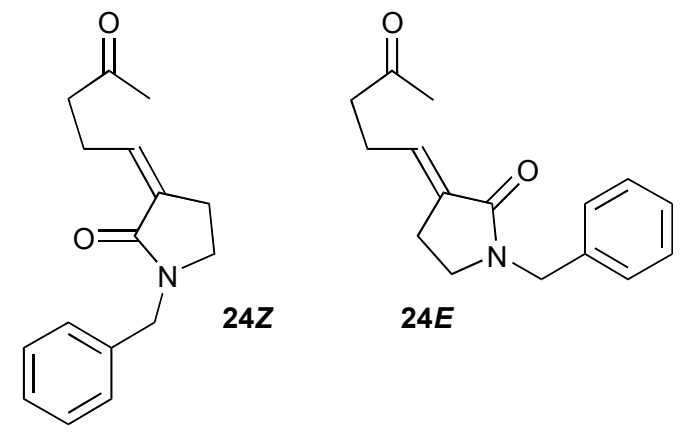

\section{(Z)-1-Benzyl-3-(4-oxo-pentylidene)-pyrrolidin-2-one $24 Z$ and (E)-1-Benzyl- 3-(4-oxo-pentylidene)-pyrrolidin-2-one 24E}

18-Crown-6 (500 mg, $1.90 \mathrm{mmol}, 5 \mathrm{eq}$ ) was added to a solution of 1-benzyl-3(diethoxyphosphinyl)-pyrrolidin-2-one 17 (118 mg, $0.38 \mathrm{mmol}, 1 \mathrm{eq})$ in THF (3 ml) at $-78^{\circ} \mathrm{C}$. The resultant solution was stirred for $5 \mathrm{~min}$ before addition of KHMDS $(0.5$ $\mathrm{M}$ in PhMe, $0.76 \mathrm{ml}, 0.38 \mathrm{mmol}, 1 \mathrm{eq})$. After $30 \mathrm{~min}$, a solution of 4-oxo-pentanal 18 (45 mg, $0.45 \mathrm{mmol}, 1.2 \mathrm{eq}$ ) in THF ( $3 \mathrm{ml}$ ) was added via cannula, and the solution was left to warm to room temperature and stir for $18 \mathrm{~h}$. Aqueous saturated $\mathrm{NH}_{4} \mathrm{Cl}(10$ $\mathrm{ml}$ ) was then added dropwise, and the aqueous layer was separated and extracted with $\mathrm{Et}_{2} \mathrm{O}(3 \times 20 \mathrm{ml})$. The combined organic extracts were washed with $\mathrm{H}_{2} \mathrm{O}$ (dist.) $(2 \times 10$ $\mathrm{ml})$, dried $\left(\mathrm{MgSO}_{4}\right)$, and concentrated in vacuo to give the crude product. Purification by flash column chromatography (eluting with $80 \%$ EtOAc in petroleum ether (40$60^{\circ} \mathrm{C}$ )) gave $(Z)$-1-benzyl-3-(4-oxo-pentylidene)-pyrrolidin-2-one $\mathbf{2 4 Z}$ (16 mg, 0.06 mmol, 16\%) as a pale yellow oil: $\square_{\max }\left(\mathrm{CDCl}_{3} \mathrm{sol}^{\mathrm{n}}\right) / \mathrm{cm}^{-1} 2255 \mathrm{~s}, 1714 \mathrm{~m}$ (ketone $\mathrm{C}=\mathrm{O}$ ), $1677 \mathrm{~m}$ (amide $\mathrm{C}=\mathrm{O}$ ), 1651m (C=C), 1440w, 1425w, 1383w, 1278w, 1162w and $1094 \mathrm{w} ; \mathrm{\square}_{\mathrm{H}}\left(400 \mathrm{MHz}, \mathrm{CDCl}_{3}\right) 2.01\left(3 \mathrm{H}, \mathrm{s}, \mathrm{C}(\mathrm{O}) \mathrm{CH}_{3}\right), 2.44\left(2 \mathrm{H}, \mathrm{t}, J\right.$ 7.1, $\left.\mathrm{CH}_{2} \mathrm{C}(\mathrm{O})\right)$, $2.48\left(2 \mathrm{H}, \mathrm{dt}, J 2.4,5.1, \mathrm{CH}=\mathrm{CCH}_{2}\right), 2.90\left(2 \mathrm{H}\right.$, apparent q, $J$ 7.2, $\left.\mathrm{CH}_{2} \mathrm{CH}=\mathrm{C}\right), 3.06$ 
$\left(2 \mathrm{H}, \mathrm{t}, J 5.1, \mathrm{CH}_{2} \mathrm{~N}\right), 4.34\left(2 \mathrm{H}, \mathrm{s}, \mathrm{CH}_{2} \mathrm{Ph}\right), 5.76(1 \mathrm{H}, \mathrm{tt}, J 2.4,7.8, \mathrm{C}=\mathrm{CH}) 7.07-7.14$ (3H, m, 3 x Ar CH) and 7.16- $7.22\left(2 \mathrm{H}, \mathrm{m}, 2\right.$ x Ar CH); $\square_{\mathrm{c}}\left(100 \mathrm{MHz}, \mathrm{CDCl}_{3}\right) 21.7$ $\left(\mathrm{CH}_{2} \mathrm{CH}=\mathrm{C}\right), 25.8\left(\mathrm{CH}=\mathrm{CCH}_{2}\right), 30.0\left(\mathrm{C}(\mathrm{O}) \mathrm{CH}_{3}\right), 44.0\left(\mathrm{CH}_{2} \mathrm{C}(\mathrm{O})\right), 44.1\left(\mathrm{CH}_{2} \mathrm{~N}\right), 47.2$ $\left(\mathrm{CH}_{2} \mathrm{Ph}\right), 127.9(\mathrm{Ar} C \mathrm{H}), 128.6$ (2 x Ar $\left.\mathrm{CH}\right), 129.1$ (2 x Ar $C H$ ), 130.8 (Ar C), 135.3 $(C H=\mathrm{C}), 136.9(\mathrm{CH}=C), 168.7(C(\mathrm{O}) \mathrm{N})$ and $209.0(C(\mathrm{O}))$.

Further elution gave (E)-1-benzyl-3-(4-oxo-pentylidene)-pyrrolidin-2-one $\mathbf{2 4 E}$ (52 $\mathrm{mg}, 0.20 \mathrm{mmol}, 53 \%)$ as a pale yellow oil: $\square_{\max }\left(\mathrm{CDCl}_{3} \mathrm{sol}^{\mathrm{n}}\right) / \mathrm{cm}^{-1} 2920 \mathrm{~m}, 1714 \mathrm{~s}$ (ketone $\mathrm{C}=\mathrm{O}$ ), 1680 (amide $\mathrm{C}=\mathrm{O})$ 1670s $(\mathrm{C}=\mathrm{C}), 1495 \mathrm{~m}, 1448 \mathrm{~m}, 1427 \mathrm{~m}, 1360 \mathrm{w}$, $1286 \mathrm{~m}, 1256 \mathrm{~m}$ and $1165 \mathrm{w}$; $\square_{\mathrm{H}}\left(400 \mathrm{MHz}, \mathrm{CDCl}_{3}\right) 2.00\left(3 \mathrm{H}, \mathrm{s}, \mathrm{C}(\mathrm{O}) \mathrm{CH}_{3}\right), 2.30(2 \mathrm{H}$, apparent q, J 7.4, $\left.\mathrm{CH}_{2} \mathrm{CH}=\mathrm{C}\right), 2.44\left(2 \mathrm{H}, \mathrm{t}, J\right.$ 7.2, $\left.\mathrm{CH}_{2} \mathrm{C}(\mathrm{O})\right), 2.52(2 \mathrm{H}, \mathrm{dt}, J$ 2.7 , 6.6, $\left.\mathrm{CH}=\mathrm{CCH}_{2}\right), 3.13\left(2 \mathrm{H}, \mathrm{t}, J\right.$ 6.6, $\left.\mathrm{CH}_{2} \mathrm{~N}\right), 4.38\left(2 \mathrm{H}, \mathrm{s}, \mathrm{CH}_{2} \mathrm{Ph}\right), 6.23(1 \mathrm{H}, \mathrm{tt}, J$ 2.7, 7.4, $\mathrm{CH}=\mathrm{C}) 7.08-7.14(3 \mathrm{H}, \mathrm{m}, 3 \mathrm{x} \mathrm{Ar} \mathrm{CH})$ and $7.15-7.19(2 \mathrm{H}, \mathrm{m}, 2 \times \mathrm{Ar} \mathrm{CH})$; $\square_{\mathrm{c}}(100$ $\left.\mathrm{MHz}, \mathrm{CDCl}_{3}\right) 22.1\left(\mathrm{CH}=\mathrm{CCH}_{2}\right), 23.6\left(\mathrm{CH}_{2} \mathrm{CH}=\mathrm{C}\right), 30.5\left(\mathrm{C}(\mathrm{O}) \mathrm{CH}_{3}\right), 42.4\left(\mathrm{CH}_{2} \mathrm{C}(\mathrm{O})\right)$, $44.1\left(\mathrm{CH}_{2} \mathrm{~N}\right), 47.5\left(\mathrm{CH}_{2} \mathrm{Ph}\right), 128.0(\mathrm{Ar} \mathrm{CH}), 128.7$ (2 x Ar $\left.\mathrm{CH}\right), 129.1(2 \times \mathrm{Ar} \mathrm{CH})$, $132.9(\mathrm{Ar} C), 131.4(C \mathrm{H}=\mathrm{C}), 136.8(\mathrm{CH}=C), 168.6(C(\mathrm{O}) \mathrm{N})$ and $207.8(C(\mathrm{O})) ; \mathrm{m} / z$ (EI mode) $257\left(\left(\mathrm{M}^{+\bullet}\right)\right.$ 45\%), 256 (1), 214 (70), 186 (20), 171 (7), 170 (5), 118 (10), 104 (4), 91 (100), 65 (12) and 43 (22); (Found: $\left(\mathrm{M}^{+\bullet}\right)$, 257.1417. $\mathrm{C}_{16} \mathrm{H}_{19} \mathrm{O}_{2} \mathrm{~N}$ requires $M, 257.1416)$.

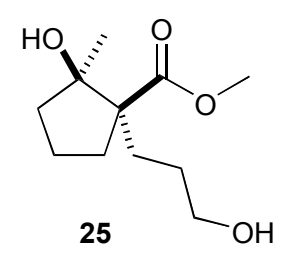

\section{rel-(1R, 2R)-Methyl-2-hydroxy-1-(3-hydroxy-propyl)-2-methyl- cyclopentanoate 25}

As for general procedure A. A 1:1 mixture of (Z)-3-(4-oxo-pentylidene)-tetrahydropyran-2-one $\mathbf{2 1 Z}$ and (E)-3-(4-oxo-pentylidene)-tetrahydro-pyran-2-one $\mathbf{2 1 E}$ (50 $\mathrm{mg}$, $0.27 \mathrm{mmol}, 1 \mathrm{eq}$ ) after a reaction time of $30 \mathrm{~min}$ and purification of the crude product mixture by column chromatography (eluting with $80 \%$ EtOAc in $\mathrm{CH}_{2} \mathrm{Cl}_{2}$ ), gave rel(1R, 2R)-methyl-2-hydroxy-1-(3-hydroxy-propyl)-2-methyl-cyclopentanoate 25 (47 $\mathrm{mg}, 0.22 \mathrm{mmol}, 81 \%$ ) as a clear, colourless oil: $\square_{\max }($ neat $) / \mathrm{cm}^{-1} 3392 \mathrm{bs}(\mathrm{OH}), 2953 \mathrm{~s}$, 
$1714 \mathrm{~s}(\mathrm{C}=\mathrm{O}), 1452 \mathrm{~m}, 1261 \mathrm{~m}, 1194 \mathrm{~m}, 1173 \mathrm{~m}, 1122 \mathrm{~m}, 1055 \mathrm{~m}$ and $1007 \mathrm{~m} ; \square_{\mathrm{H}}(400$ $\left.\mathrm{MHz}, \mathrm{CDCl}_{3}\right) 1.22-1.38\left(2 \mathrm{H}, \mathrm{m}, 1 \mathrm{H}\right.$ from $\mathrm{CH}_{2} \mathrm{CH}_{2} \mathrm{OH}, 1 \mathrm{H}$ from $\left.\mathrm{CH}_{2}\right), 1.33(3 \mathrm{H}, \mathrm{s}$, $\left.\mathrm{CH}_{3}\right), 1.45-1.57\left(2 \mathrm{H}, \mathrm{m}, 1 \mathrm{H}\right.$ from $\mathrm{CH}_{2} \mathrm{CH}_{2} \mathrm{OH}, 1 \mathrm{H}$ from $\left.\mathrm{CH}_{2}\right), 1.59-1.66(1 \mathrm{H}, \mathrm{m}$, $1 \mathrm{H}$ from $\left.\mathrm{CH}_{2} \mathrm{C}(\mathrm{OH})\right), 1.69-1.82\left(4 \mathrm{H}, \mathrm{m}, 4 \mathrm{H}\right.$ from $\left.\mathrm{CH}_{2}\right), 2.35-2.42(1 \mathrm{H}, \mathrm{m}, 1 \mathrm{H}$ from $\left.\mathrm{CH}_{2} \mathrm{C}(\mathrm{OH})\right), 3.55-3.61\left(2 \mathrm{H}, \mathrm{m}, \mathrm{CH}_{2} \mathrm{O}\right)$ and $3.65\left(3 \mathrm{H}, \mathrm{s}, \mathrm{OCH}_{3}\right)$; $\square_{\mathrm{c}}(100 \mathrm{MHz}$, $\left.\mathrm{CDCl}_{3}\right) 20.1\left(\mathrm{CH}_{2}\right), 23.6\left(\mathrm{C}(\mathrm{OH}) \mathrm{CH}_{3}\right), 29.2\left(\mathrm{CH}_{2}\right), 31.0\left(\mathrm{CH}_{2}\right), 31.6\left(\mathrm{CH}_{2} \mathrm{C}(\mathrm{OH})\right)$, $40.1\left(\mathrm{CH}_{2}\right), 52.2\left(\mathrm{OCH}_{3}\right), 60.5(\mathrm{C}), 63.4\left(\mathrm{CH}_{2} \mathrm{O}\right), 83.4(\mathrm{C}(\mathrm{OH}))$ and $176.6(\mathrm{C}(\mathrm{O})) ; \mathrm{m} / \mathrm{z}$ (CI mode, isobutane) $217\left((\mathrm{M}+\mathrm{H})^{+} 25 \%\right), 199$ (100), 185 (40), 171 (35), 167 (30), 156 (5), 139 (10), 113 (10), 97 (15) and 85 (25); (Found: $(\mathrm{M}+\mathrm{H})^{+}, 217.1438$. $\mathrm{C}_{11} \mathrm{H}_{21} \mathrm{O}_{4}$ requires $\left.M, 217.1440\right)$.

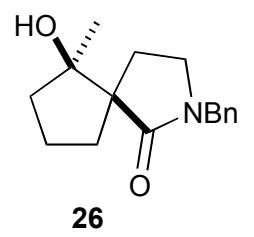

rel-(5R, 6R)-2-Benzyl-6-hydroxy-6-methyl-2-aza-spiro[4.4]nonan-1-one 26

As for general procedure A. (E)-1-Benzyl-3-(4-oxo-pentylidene)-pyrrolidin-2-one $\mathbf{2 4 E}$ (50 $\mathrm{mg}, 0.19 \mathrm{mmol}, 1 \mathrm{eq}$ ) after a reaction time of $3 \mathrm{~h}$ and purification of the crude product mixture by column chromatography (eluting with $10 \%$ EtOAc in petroleum ether $\left.\left(40-60^{\circ} \mathrm{C}\right)\right)$, gave $r$ el-(5R, 6R)-2-benzyl-6-hydroxy-6-methyl-2-azaspiro[4.4]nonan-1-one 26 (4.60 mg, $0.02 \mathrm{mmol}$, 9\%) as a clear, colourless oil: $\square_{\max }$ $\left(\mathrm{CDCl}_{3} \mathrm{sol}^{\mathrm{n}}\right) / \mathrm{cm}^{-1} 3012 \mathrm{~s}(\mathrm{OH}), 2397 \mathrm{~m}, 2256 \mathrm{w}, 1661 \mathrm{w}(\mathrm{C}=\mathrm{O}), 1598 \mathrm{w}, 1525 \mathrm{w}, 1483 \mathrm{w}$, $1420 \mathrm{w}, 1382 \mathrm{w}, 1215 \mathrm{~s}$ and $1098 \mathrm{w} ; \square_{\mathrm{H}}\left(400 \mathrm{MHz} \mathrm{CDCl}_{3}\right) 1.17\left(3 \mathrm{H}, \mathrm{s}, \mathrm{C}(\mathrm{OH}) \mathrm{CH}_{3}\right)$, $1.50-1.67\left(4 \mathrm{H}, \mathrm{m}, 1 \mathrm{H}\right.$ from $\mathrm{CH}_{2} \mathrm{CC}(\mathrm{OH}), 1 \mathrm{H}$ from $\mathrm{CH}_{2} \mathrm{C}(\mathrm{OH}), 1 \mathrm{H}$ from $\mathrm{CH}_{2} \mathrm{CH}_{2} \mathrm{C}(\mathrm{OH}), 1 \mathrm{H}$ from $\left.\mathrm{NC}(\mathrm{O}) \mathrm{CCH}_{2}\right), 1.82-1.96\left(3 \mathrm{H}, \mathrm{m}, 1 \mathrm{H}\right.$ from $\mathrm{CH}_{2} \mathrm{CC}(\mathrm{OH})$, $1 \mathrm{H}$ from $\mathrm{CH}_{2} \mathrm{CH}_{2} \mathrm{C}(\mathrm{OH}), 1 \mathrm{H}$ from $\left.\mathrm{NC}(\mathrm{O}) \mathrm{CCH}_{2}\right), 2.25-2.31(1 \mathrm{H}, \mathrm{m}, 1 \mathrm{H}$ from $\left.\mathrm{CH}_{2} \mathrm{C}(\mathrm{OH})\right), 3.04-3.16\left(2 \mathrm{H}, \mathrm{m}, \mathrm{NCH}_{2}\right), 4.36(1 \mathrm{H}$, apparent d, $J$ 14.6, AB system, $1 \mathrm{H}$ from $\left.\mathrm{CH}_{2} \mathrm{Ph}\right), 4.47\left(1 \mathrm{H}\right.$, apparent d, $J$ 14.6, $\mathrm{AB}$ system, $1 \mathrm{H}$ from $\left.\mathrm{CH}_{2} \mathrm{Ph}\right), 4.97(1 \mathrm{H}, \mathrm{d}$, $J$ 1.5, $\mathrm{OH}), 7.15-7.22(3 \mathrm{H}, \mathrm{m}, 3 \times \mathrm{Ar} \mathrm{CH})$ and $7.23-7.29(2 \mathrm{H}, \mathrm{m}, 2 \times \mathrm{Ar} \mathrm{CH})$; $\square_{\mathrm{c}}$ $\left(100 \mathrm{MHz}, \mathrm{CDCl}_{3}\right) 20.7\left(\mathrm{CH}_{2} \mathrm{CH}_{2} \mathrm{C}(\mathrm{OH})\right), 23.0\left(\mathrm{C}(\mathrm{OH}) \mathrm{CH}_{3}\right), 29.6\left(\mathrm{CH}_{2} \mathrm{CC}(\mathrm{O}) \mathrm{N}\right)$, $34.4\left(\mathrm{CH}_{2} \mathrm{C}(\mathrm{OH})\right), 39.0\left(\mathrm{CH}_{2} \mathrm{CC}(\mathrm{OH})\right), 44.0\left(\mathrm{C}(\mathrm{O}) \mathrm{NCH}_{2}\right), 47.1\left(\mathrm{CH}_{2} \mathrm{Ph}\right), 56.2(C)$, $82.9(\mathrm{C}(\mathrm{OH})), 128.1(\mathrm{Ar} C \mathrm{H}), 128.4(2 \times \mathrm{Ar} C \mathrm{H}), 129.2(2 \times \mathrm{Ar} C \mathrm{H}), 136.5(\operatorname{Ar} C)$ 
and $178.4(C(\mathrm{O}) \mathrm{N}) ; \mathrm{m} / \mathrm{z}\left(\mathrm{EI}\right.$ mode) $259\left(\left(\mathrm{M}^{+\bullet}\right) 20 \%\right), 241(5), 216(5), 201(2), 188$ (50), 175 (5), 149 (2), 121 (3), 114 (7), 84 (100), 83 (65) and 47 (30); (Found: (M+•), 259.1571. $\mathrm{C}_{16} \mathrm{H}_{21} \mathrm{O}_{2} \mathrm{~N}$ requires $M, 259.1572$ ).
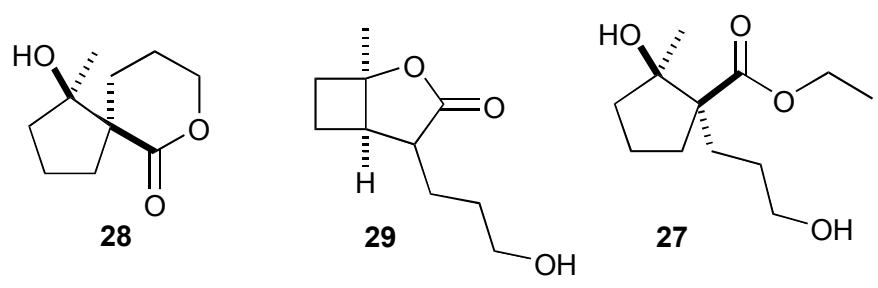

rel-(1R, 5R)-1-Hydroxy-1-methyl-7-oxa-spiro[4.5]decan-6-one 28 and rel(1R, 4R/S, 5R)-4-(3-Hydroxy-propyl)-1-methyl-2-oxa-bicyclo[3.2.0]heptan3-one 29 and rel-(1R, 2R)-Ethyl-2-hydroxy-1-(3-hydroxy-propyl)-2-methylcyclopentanoate 27

Dry EtOH (3 ml) was added to a stirred solution of $\operatorname{SmI}_{2}(0.1 \mathrm{M}$ in THF, $11 \mathrm{ml}, 1.10$ mmol, 4 eq) at $0^{\circ} \mathrm{C}$ and the resulting solution was stirred for $10 \mathrm{~min}$. A 2.5:1 mixture of (Z )-3-(4-oxo-pentylidene)-tetrahydro-pyran-2-one $\mathbf{2 1 Z}$ and (E)-3-(4-oxopentylidene)-tetrahydro-pyran-2-one $\mathbf{2 1 E}(50 \mathrm{mg}, 0.27 \mathrm{mmol}, 1 \mathrm{eq})$ in THF (1 ml) was added, and the resultant solution stirred at $0^{\circ} \mathrm{C}$ for $40 \mathrm{~min}$. The reaction was quenched by opening to the air, followed by the addition of aqueous saturated $\mathrm{NaCl}$ $(10 \mathrm{ml})$. The aqueous layer was separated and extracted with EtOAc. The combined organic extracts were dried $\left(\mathrm{MgSO}_{4}\right)$ and concentrated in vacuo to give the crude product. Purification by column chromatography (eluting with $30 \%$ EtOAc in $\left.\mathrm{CH}_{2} \mathrm{Cl}_{2}\right)$, gave rel-(1R, 5R)-1-hydroxy-1-methyl-7-oxa-spiro[4.5]decan-6-one 28 (6 $\mathrm{mg}, 0.03 \mathrm{mmol}, 12 \%$ ) as a pale yellow oil: $\square_{\max }($ neat $) / \mathrm{cm}^{-1} 3417 \mathrm{bs}(\mathrm{OH}), 2962 \mathrm{~s}$, 1714s $(\mathrm{C}=\mathrm{O}), 1450 \mathrm{~m}, 1394 \mathrm{~m}, 1263 \mathrm{~m}, 1146 \mathrm{~s}$ and 962s; $\square_{\mathrm{H}}\left(400 \mathrm{MHz}, \mathrm{CDCl}_{3}\right) 1.19$ $\left(3 \mathrm{H}, \mathrm{s}, \mathrm{CH}_{3}\right), 1.55-1.66\left(3 \mathrm{H}, \mathrm{m}, 3 \mathrm{H}\right.$ from $\left.\mathrm{CH}_{2}\right), 1.75-1.95(6 \mathrm{H}, \mathrm{m}, 1 \mathrm{H}$ from $\mathrm{CH}_{2} \mathrm{C}(\mathrm{OH}), 5 \mathrm{H}$ from $\left.\mathrm{CH}_{2}\right), 2.23-2.28\left(1 \mathrm{H}, \mathrm{m}, 1 \mathrm{H}\right.$ from $\left.\mathrm{CH}_{2} \mathrm{C}(\mathrm{OH})\right), 4.22-4.29$ $\left(1 \mathrm{H}, \mathrm{m}, 1 \mathrm{H}\right.$ from $\left.\mathrm{CH}_{2} \mathrm{O}\right), 4.33-4.39\left(1 \mathrm{H}, \mathrm{m}, 1 \mathrm{H}\right.$ from $\left.\mathrm{CH}_{2} \mathrm{O}\right)$ and $4.65(1 \mathrm{H}, \mathrm{s}, \mathrm{OH})$; $\square_{\mathrm{C}}\left(100 \mathrm{MHz}, \mathrm{CDCl}_{3}\right) 19.1\left(\mathrm{CH}_{2}\right), 19.8\left(\mathrm{CH}_{2}\right), 21.7\left(\mathrm{CH}_{3}\right), 26.8\left(\mathrm{CH}_{2}\right), 34.8$ $\left(\mathrm{CH}_{2} \mathrm{C}(\mathrm{OH})\right), 37.1\left(\mathrm{CH}_{2}\right), 53.8(\mathrm{C}), 68.8\left(\mathrm{CH}_{2} \mathrm{O}\right), 82.4(\mathrm{C}(\mathrm{OH}))$ and $176.5(\mathrm{C}(\mathrm{O})) ; \mathrm{m} / \mathrm{z}$ (EI mode) $184\left(\left(\mathrm{M}^{+*}\right)\right.$ 5\%), 166 (5), 151 (2), 126 (30), 113 (100), 84 (45), 83 (70), 67 (20) and 43 (40); (Found: $\left(\mathrm{M}^{+\bullet}\right), 184.1099 . \mathrm{C}_{10} \mathrm{H}_{16} \mathrm{O}_{3}$ requires $\left.M, 184.1099\right)$. 
Further elution then gave rel-(1R, 4R/S, 5R)-4-(3-hydroxy-propyl)-1-methyl-2-oxabicyclo[3.2.0]heptan-3-one 29 (5 $\mathrm{mg}, 0.03 \mathrm{mmol}, 10 \%)$ as a clear, colourless oil:

Characterised as a single diastereoisomer

$\square_{\max }\left(\mathrm{CDCl}_{3} \mathrm{sol}^{\mathrm{n}}\right) / \mathrm{cm}^{-1} 3011 \mathrm{~s}(\mathrm{OH}), 2402 \mathrm{~m}, 1755 \mathrm{~m}(\mathrm{C}=\mathrm{O}), 1514 \mathrm{w}, 1420 \mathrm{w}, 1225 \mathrm{~s}$ and $1015 \mathrm{w} ; \square_{\mathrm{H}}\left(400 \mathrm{MHz}, \mathrm{CDCl}_{3}\right) 1.42\left(3 \mathrm{H}, \mathrm{s}, \mathrm{CCH}_{3}\right), 1.49-1.66(4 \mathrm{H}, \mathrm{m}, 1 \mathrm{H}$ from $\mathrm{CH}_{2} \mathrm{CH}_{2} \mathrm{CH}_{2} \mathrm{OH}, 2 \mathrm{H}$ from $\mathrm{CH}_{2} \mathrm{CH}_{2} \mathrm{OH}, 1 \mathrm{H}$ from $\left.\mathrm{CCH}_{2} \mathrm{CH}_{2} \mathrm{CH}\right), 1.73-1.78(1 \mathrm{H}, \mathrm{m}$, $1 \mathrm{H}$ from $\left.\mathrm{CH}_{2} \mathrm{CH}_{2} \mathrm{CH}_{2} \mathrm{OH}\right), 2.10-2.20\left(3 \mathrm{H}, \mathrm{m}, 3 \mathrm{H}\right.$ from $\left.\mathrm{CCH}_{2} \mathrm{CH}_{2} \mathrm{CH}\right), 2.45-2.47$ $\left(1 \mathrm{H}, \mathrm{m}, \mathrm{CH}_{2} \mathrm{CHCH}\right), 2.54(1 \mathrm{H}, \mathrm{dt}, J$ 2.7, 5.9, $\mathrm{CHC}(\mathrm{O}))$ and $3.61(2 \mathrm{H}, \mathrm{t}, J$ 5.7, $\left.\mathrm{CH}_{2} \mathrm{OH}\right) ; \square_{\mathrm{c}}\left(100 \mathrm{MHz}, \mathrm{CDCl}_{3}\right) 21.4\left(\mathrm{CH}_{2}\right), 24.8\left(\mathrm{CCH}_{3}\right), 29.1\left(\mathrm{CH}_{2} \mathrm{CH}_{2} \mathrm{CH}_{2} \mathrm{OH}\right)$, $30.7\left(\mathrm{CH}_{2} \mathrm{CH}_{2} \mathrm{OH}\right), 33.5\left(\mathrm{CH}_{2}\right), 45.1\left(\mathrm{CH}_{2} \mathrm{CHCH}\right), 49.6(\mathrm{CHC}(\mathrm{O})), 62.7\left(\mathrm{CH}_{2} \mathrm{OH}\right)$, $87.0\left(C\left(\mathrm{CH}_{3}\right) \mathrm{O}\right)$ and $180.6(\mathrm{C}(\mathrm{O})) ; \mathrm{m} / \mathrm{z}(\mathrm{CI}$ mode, isobutane $) 185\left((\mathrm{M}+\mathrm{H})^{+} 100\right), 167$ (15), 139 (2), 129 (10), 97 (3), 85 (85) and 71 (7); (Found: $(\mathrm{M}+\mathrm{H})^{+}, 185.1177$. $\mathrm{C}_{10} \mathrm{H}_{17} \mathrm{O}_{3}$ requires $M, 185.1178$ ).

Further elution then gave rel-(1R, 2R)-ethyl-2-hydroxy-1-(3-hydroxy-propyl)-2methyl-cyclopentanoate 27 (20 mg, $0.09 \mathrm{mmol}, 41 \%)$ as a clear, colourless oil: $\square_{\max }$ (neat) $/ \mathrm{cm}^{-1}$ 3406bs $(\mathrm{OH}), 2958 \mathrm{~m}, 1709 \mathrm{~s}(\mathrm{C}=\mathrm{O}), 1446 \mathrm{~m}, 1369 \mathrm{~m}, 1265 \mathrm{~m}, 1180 \mathrm{~m}$, $1122 \mathrm{~m}, 1055 \mathrm{~m}$ and 1030m; $\square_{\mathrm{H}}\left(400 \mathrm{MHz}, \mathrm{CDCl}_{3}\right) 1.22\left(3 \mathrm{H}, \mathrm{t}, J\right.$ 7.1, $\left.\mathrm{CH}_{2} \mathrm{CH}_{3}\right), 1.22-$ $1.29\left(1 \mathrm{H}, \mathrm{m}, 1 \mathrm{H}\right.$ from $\left.\mathrm{CH}_{2}\right), 1.30\left(3 \mathrm{H}, \mathrm{s}, \mathrm{C}(\mathrm{OH}) \mathrm{CH}_{3}\right), 1.37-1.42(1 \mathrm{H}, \mathrm{m}, 1 \mathrm{H}$ from $\left.\mathrm{CH}_{2}\right), 1.47-1.53\left(2 \mathrm{H}, \mathrm{m}, \mathrm{CH}_{2}\right), 1.58-1.79\left(5 \mathrm{H}, \mathrm{m}, 1 \mathrm{H}\right.$ from $\mathrm{CH}_{2} \mathrm{C}(\mathrm{OH}) 4 \mathrm{H}$ from $\left.\mathrm{CH}_{2}\right), 2.33-2.41\left(1 \mathrm{H}, \mathrm{m}, 1 \mathrm{H}\right.$ from $\left.\mathrm{CH}_{2} \mathrm{C}(\mathrm{OH})\right), 3.50-3.60\left(2 \mathrm{H}, \mathrm{m}, \mathrm{CH}_{2} \mathrm{OH}\right)$ and $4.11\left(2 \mathrm{H}, \mathrm{q}, J\right.$ 7.1, $\left.\mathrm{OCH}_{2} \mathrm{CH}_{3}\right)$; $\square_{\mathrm{c}}\left(100 \mathrm{MHz}, \mathrm{CDCl}_{3}\right) 14.7\left(\mathrm{OCH}_{2} \mathrm{CH}_{3}\right), 20.0\left(\mathrm{CH}_{2}\right)$, $23.6\left(\mathrm{C}(\mathrm{OH}) \mathrm{CH}_{3}\right), 29.2\left(\mathrm{CH}_{2}\right), 30.9\left(\mathrm{CH}_{2}\right), 31.6\left(\mathrm{CH}_{2} \mathrm{C}(\mathrm{OH})\right), 40.1\left(\mathrm{CH}_{2}\right), 60.3$ $(C \mathrm{C}(\mathrm{O})), 61.0\left(\mathrm{OCH}_{2} \mathrm{CH}_{3}\right), 63.4\left(\mathrm{CH}_{2} \mathrm{OH}\right), 83.3(\mathrm{C}(\mathrm{OH}))$ and $176.1(\mathrm{C}(\mathrm{O})) ; \mathrm{m} / \mathrm{z}(\mathrm{CI}$ mode, isobutane) $231\left((\mathrm{M}+\mathrm{H})^{+} 100\right), 213$ (100), 185 (70), 167 (90), 139 (30), 113 (5), 99 (5), 85 (5) and 81 (2); (Found: $(\mathrm{M}+\mathrm{H})^{+}, 231.1585 . \mathrm{C}_{12} \mathrm{H}_{23} \mathrm{O}_{4}$ requires $M$, 231.1596). 


\section{General Cyclobutane-forming Procedure D}

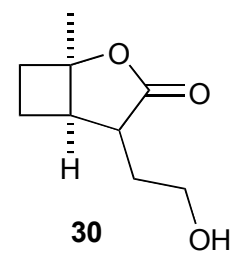

rel-(1R, 4R/S, 5R)-4-(2-Hydroxy-ethyl)-1-methyl-2-oxabicyclo[3.2.0]heptan-3-one 30

Dry ${ }^{\mathrm{t}} \mathrm{BuOH}(6.5 \mathrm{ml})$ was added to a stirred solution of $\mathrm{SmI}_{2}(0.1 \mathrm{M}$ in THF, $24 \mathrm{ml}$, $2.40 \mathrm{mmol}, 4 \mathrm{eq})$ at $0^{\circ} \mathrm{C}$ and the resulting solution was stirred for $10 \mathrm{~min}$. (E)-3-(4Oxo-pentylidene)-dihydro-furan-2-one 6 (100 mg, $0.60 \mathrm{mmol}, 1 \mathrm{eq})$ in THF (2 ml) was added, and the resultant solution stirred at $0^{\circ} \mathrm{C}$ for $4 \mathrm{~h}$. The reaction was quenched by opening to the air, followed by the addition of aqueous saturated $\mathrm{NaCl}$ $(10 \mathrm{ml})$. The aqueous layer was separated and extracted with $80 \%$ EtOAc in petroleum ether $\left(40-60^{\circ} \mathrm{C}\right)(4 \times 15 \mathrm{ml})$. The combined organic extracts were dried $\left(\mathrm{MgSO}_{4}\right)$ and concentrated in vacuo to give the crude product. Purification by column chromatography (eluting first with $80 \%$ EtOAc in petroleum ether $\left(40-60^{\circ} \mathrm{C}\right)$, then with $30 \% \mathrm{Et}_{2} \mathrm{O}$ in $\mathrm{CHCl}_{3}$ ), gave rel-(1R, 4R/S, 5R)-4-(2-hydroxy-ethyl)-1-methyl-2oxa-bicyclo[3.2.0]heptan-3-one 30 (65 mg, $0.38 \mathrm{mmol}, 64 \%)$ as a clear, colourless oil:

\section{Characterised as a mixture of diastereoisomers}

$\square_{\max }($ neat $) / \mathrm{cm}^{-1} 3406 \mathrm{bs}(\mathrm{OH}), 2941 \mathrm{~m}, 1745 \mathrm{~s}(\mathrm{C}=\mathrm{O}), 1383 \mathrm{~m}, 1250 \mathrm{~m}, 1174 \mathrm{~m}, 1122 \mathrm{~m}$, $1057 \mathrm{~m}$ and 949m; $\square_{\mathrm{H}}\left(400 \mathrm{MHz}, \mathrm{CDCl}_{3}\right) 1.37\left(3 \mathrm{H}, \mathrm{s}, \mathrm{CH}_{3}\right.$ from minor), $1.43(3 \mathrm{H}, \mathrm{s}$, $\mathrm{CH}_{3}$ from major), $1.64-2.23\left(12 \mathrm{H}, \mathrm{m}, 3 \times \mathrm{CH}_{2}\right.$ from minor, $3 \times \mathrm{CH}_{2}$ from major), $2.48-2.50\left(1 \mathrm{H}, \mathrm{m}, \mathrm{CH}_{2} \mathrm{CHCHC}(\mathrm{O})\right.$ from major $), 2.70(1 \mathrm{H}, \mathrm{dt}, J$ 2.8, 7.5, $\mathrm{CH}_{2} \mathrm{CHCHC}(\mathrm{O})$ from major), $2.79-2.91(2 \mathrm{H}, \mathrm{m}, 2 \times \mathrm{CH}$ from minor) and $3.60-$ 3.77 (4H, m, $\mathrm{CH}_{2} \mathrm{OH}$ from minor, $\mathrm{CH}_{2} \mathrm{OH}$ from major); $\square_{\mathrm{C}}\left(100 \mathrm{MHz}, \mathrm{CDCl}_{3}\right) 17.2$ ( $\mathrm{CH}_{2}$ from minor), $21.5\left(\mathrm{CH}_{2}\right.$ from major $), 23.7\left(\mathrm{CH}_{3}\right.$ from minor $\left.),\right), 24.8\left(\mathrm{CH}_{3}\right.$ from major), $29.0\left(\mathrm{CH}_{2}\right.$ from minor), $32.2\left(\mathrm{CH}_{2}\right.$ from major), $33.4\left(\mathrm{CH}_{2}\right.$ from minor $), 35.3$ $\left(\mathrm{CH}_{2}\right.$ from major), 42.3 ( $\mathrm{CH}$ from minor), 43.2 ( $\mathrm{CH}$ from minor), 45.2 ( $\mathrm{CHCHC}(\mathrm{O})$ from major), $47.2\left(\mathrm{CHC} \mathrm{HC}(\mathrm{O})\right.$ from major), $60.9\left(\mathrm{CH}_{2} \mathrm{OH}\right.$ from major), 61.7 
$\left(\mathrm{CH}_{2} \mathrm{OH}\right.$ from minor), $87.5\left(\mathrm{C}\left(\mathrm{CH}_{3}\right) \mathrm{O}\right.$ from major $), 88.1\left(\mathrm{C}\left(\mathrm{CH}_{3}\right) \mathrm{O}\right.$ from minor), $180.1(C(\mathrm{O})$ from minor) and $181.2(C(\mathrm{O})$ from major); $\mathrm{m} / \mathrm{z}$ (CI mode, isobutane) 341 (35\%), 209 (15), $171\left((\mathrm{M}+\mathrm{H})^{+}\right.$100), 153 (70), 115 (20) and 109 (5); (Found: $(\mathrm{M}+$ $\mathrm{H})^{+}, 171.1021 . \mathrm{C}_{9} \mathrm{H}_{15} \mathrm{O}_{3}$ requires $\left.M, 171.1021\right)$.

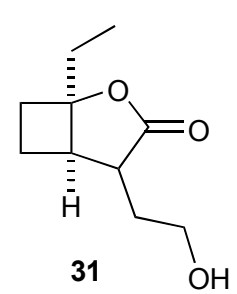

rel-(1R, 4R/S, 5R)-1-Ethyl-4-(2-hydroxy-ethyl)-2-oxa-bicyclo[3.2.0]heptan3-one 31

As for general procedure D. (E)-3-(4-Oxo-hexylidene)-dihydro-furan-2-one 7 (40 mg, $0.22 \mathrm{mmol}, 1 \mathrm{eq}$ ), after reaction time of $1 \mathrm{~h}$ and purification of the crude product mixture by column chromatography (eluting with $60 \%$ EtOAc in petroleum ether (40$\left.\left.60^{\circ} \mathrm{C}\right)\right)$ gave rel-(1R, 4R/S, 5R)-1-ethyl-4-(2-hydroxy-ethyl)-2-oxabicyclo[3.2.0]heptan-3-one 31 (12 $\mathrm{mg}, 0.07 \mathrm{mmol}, 31 \%)$ as a clear, colourless oil:

Characterised as a mixture of diastereoisomers

$\square_{\max }($ neat $) / \mathrm{cm}^{-1} 3406 \mathrm{bs}(\mathrm{OH}), 2941 \mathrm{~m}, 1743 \mathrm{~s}(\mathrm{C}=\mathrm{O}), 1462 \mathrm{~m}, 1209 \mathrm{~m}, 1132 \mathrm{~m}, 1115 \mathrm{~m}$, $1024 \mathrm{~m}$ and $943 \mathrm{~m} ; \square_{\mathrm{H}}\left(400 \mathrm{MHz}, \mathrm{CDCl}_{3}\right) 0.86\left(3 \mathrm{H}, \mathrm{t}, J\right.$ 7.4, $\mathrm{CH}_{3}$ from minor), 0.88 (3H, t, J 7.4, $\mathrm{CH}_{3}$ from major), $1.61-2.19\left(16 \mathrm{H}, \mathrm{m}, 4 \times \mathrm{CH}_{2}\right.$ from minor, 4 x $\mathrm{CH}_{2}$ from major), 2.47 - $2.50(1 \mathrm{H}, \mathrm{m}, \mathrm{CHCHC}(\mathrm{O})$ from major), $2.71(1 \mathrm{H}, \mathrm{dt}, J 3.0,7.6$, $\mathrm{CHCHC}(\mathrm{O})$ from major), $2.81-2.88$ (1H, m, $\mathrm{CHCHC}(\mathrm{O})$ from minor), $2.91-2.98$ $\left(1 \mathrm{H}, \mathrm{m}, \mathrm{CHCHC}(\mathrm{O})\right.$ from minor) and $3.58-3.77\left(4 \mathrm{H}, \mathrm{m}, \mathrm{CH}_{2} \mathrm{OH}\right.$ from minor, $\mathrm{CH}_{2} \mathrm{OH}$ from major); $\square_{\mathrm{c}}\left(100 \mathrm{MHz}, \mathrm{CDCl}_{3}\right) 7.9\left(\mathrm{CH}_{3}\right.$ from minor $), 8.0\left(\mathrm{CH}_{3}\right.$ from major), $16.9\left(\mathrm{CH}_{2}\right.$ from minor), $21.8\left(\mathrm{CH}_{2}\right.$ from major $), 29.2\left(\mathrm{CH}_{2}\right.$ from minor $), 30.3$ $\left(\mathrm{CH}_{2}\right.$ from minor), $30.4\left(\mathrm{CH}_{2}\right.$ from minor $), 30.6\left(\mathrm{CH}_{2}\right.$ from major $), 31.5\left(\mathrm{CH}_{2}\right.$ from major), $35.3\left(\mathrm{CH}_{2}\right.$ from major), 41.4 ( $\mathrm{CHCHC}(\mathrm{O})$ from minor), 42.8 ( $\mathrm{CHCHC}(\mathrm{O})$ from minor), 43.3 ( $\mathrm{CHCHC}(\mathrm{O})$ from major), 47.2 ( $\mathrm{CHCHC}(\mathrm{O})$ from major), 61.0 $\left(\mathrm{CH}_{2} \mathrm{OH}\right.$ from major), $61.7\left(\mathrm{CH}_{2} \mathrm{OH}\right.$ from minor $), 90.3\left(\mathrm{C}\left(\mathrm{CH}_{2} \mathrm{CH}_{3}\right) \mathrm{O}\right.$ from major $)$, 
$91.2\left(\mathrm{C}\left(\mathrm{CH}_{2} \mathrm{CH}_{3}\right) \mathrm{O}\right.$ from minor $), 180.4(\mathrm{C}(\mathrm{O})$ from minor $)$ and $181.5(\mathrm{C}(\mathrm{O})$ from major); $m / z$ (CI mode, isobutane) $223(5 \%), 199(15), 185\left((\mathrm{M}+\mathrm{H})^{+} 100\right), 183(50)$, 167 (20), 115 (15), 113 (2) and 81 (5); (Found: $(\mathrm{M}+\mathrm{H})^{+}, 185.1179 . \mathrm{C}_{10} \mathrm{H}_{17} \mathrm{O}_{3}$ requires $M, 185.1178)$.

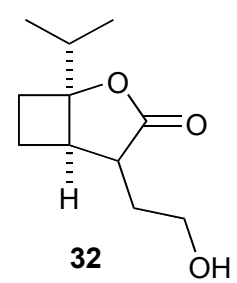

rel-(1R, 4R/S, 5R)-4-(2-Hydroxy-ethyl)-1-isopropyl-2-oxabicyclo[3.2.0]heptan-3-one 32

As for general procedure D. (E)-3-(5-methyl-4-oxo-hexylidene)-dihydro-furan-2-one $8(50 \mathrm{mg}, 0.26 \mathrm{mmol}, 1 \mathrm{eq})$, after reaction time of $1 \mathrm{~h}$ and purification of the crude product mixture by column chromatography (eluting with 50\% EtOAc in petroleum ether $\left(40-60^{\circ} \mathrm{C}\right)$ ) gave $\mathrm{rel}-(1 \mathrm{R}, 4 \mathrm{R} / \mathrm{S}, 5 \mathrm{R})-4-(2-h y d r o x y-e t h y l)-1-i s o p r o p y l-2-o x a-$ bicyclo[3.2.0]heptan-3-one 32 (28 $\mathrm{mg}, 0.14 \mathrm{mmol}, 54 \%)$ as a clear, colourless oil:

Characterised as a mixture of diastereoisomers

$\square_{\max }\left(\right.$ neat) $/ \mathrm{cm}^{-1} 3410 \mathrm{bs}(\mathrm{OH}), 2962 \mathrm{~m}, 1743 \mathrm{~s}(\mathrm{C}=\mathrm{O}), 1470 \mathrm{~m}, 1236 \mathrm{~m}, 1209 \mathrm{~m}, 1167 \mathrm{~m}$, $1053 \mathrm{~m}$ and $947 \mathrm{~m}$; $\square_{\mathrm{H}}\left(400 \mathrm{MHz}, \mathrm{CDCl}_{3}\right) 0.83\left(3 \mathrm{H}, \mathrm{d}, J 6.9, \mathrm{CH}_{3}\right), 0.85(3 \mathrm{H}, \mathrm{d}, J$ 6.9, $\left.\mathrm{CH}_{3}\right), 0.87\left(3 \mathrm{H}, \mathrm{d}, J 6.9, \mathrm{CH}_{3}\right), 0.91\left(3 \mathrm{H}, \mathrm{d}, J 6.9, \mathrm{CH}_{3}\right), 1.58-2.22\left(14 \mathrm{H}, \mathrm{m}, 3 \times \mathrm{CH}_{2}\right.$ from minor, $3 \times \mathrm{CH}_{2}$ from major, $\mathrm{CH}\left(\mathrm{CH}_{3}\right)_{2}$ from minor, $\mathrm{CH}\left(\mathrm{CH}_{3}\right)_{2}$ from major), 2.52 -2.54 (1H, m, CH from minor), $2.70-2.73(1 \mathrm{H}, \mathrm{m}, \mathrm{CH}$ from minor), 2.81 (1H, dt, $J$ 1.7, 8.1, $\mathrm{CH}$ from major), $2.97(1 \mathrm{H}$, apparent $\mathrm{q}, J$ 8.1, $\mathrm{CH}$ from major) and $3.58-3.79$ (4H, m, $\mathrm{CH}_{2} \mathrm{OH}$ from minor, $\mathrm{CH}_{2} \mathrm{OH}$ from major); $\square_{\mathrm{c}}\left(100 \mathrm{MHz}, \mathrm{CDCl}_{3}\right) 14.8\left(\mathrm{CH}_{3}\right)$, $14.9\left(\mathrm{CH}_{3}\right), 14.9\left(\mathrm{CH}_{3}\right), 15.0\left(\mathrm{CH}_{2}\right), 15.2\left(\mathrm{CH}_{3}\right), 20.4\left(\mathrm{CH}_{2}\right), 27.7\left(\mathrm{CH}_{2}\right), 27.9\left(\mathrm{CH}_{2}\right)$, $28.6\left(\mathrm{CH}_{2}\right), 32.5\left(\mathrm{CH}\left(\mathrm{CH}_{3}\right)_{2}\right), 33.5\left(\mathrm{CH}\left(\mathrm{CH}_{3}\right)_{2}\right), 33.9\left(\mathrm{CH}_{2}\right), 39.1$ ( $\mathrm{CH}$ from major), 40.5 ( $\mathrm{CH}$ from minor), 41.5 ( $\mathrm{CH}$ from major), 46.0 ( $\mathrm{CH}$ from minor), $59.7\left(\mathrm{CH}_{2} \mathrm{OH}\right.$ from minor), $60.2\left(\mathrm{CH}_{2} \mathrm{OH}\right.$ from major), 91.3 ( $\mathrm{CCH}$ from minor), 92.2 ( $\mathrm{CCH}$ from major). $179.3(C(\mathrm{O})$ from major) and $180.2(C(\mathrm{O})$ from minor); $m / z$ (CI mode, 
isobutane) $397(20 \%), 237(15), 199\left((\mathrm{M}+\mathrm{H})^{+} 100\right), 181(45), 153$ (15) and 95 (35); (Found: $(\mathrm{M}+\mathrm{H})^{+}, 199.1336 . \mathrm{C}_{11} \mathrm{H}_{19} \mathrm{O}_{3}$ requires $\left.M, 199.1334\right)$.

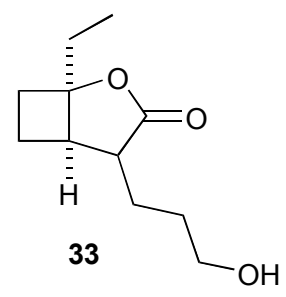

rel-(1R, 4R/S, 5R)-1-Ethyl-4-(3-hydroxy-propyl)-2-oxabicyclo[3.2.0]heptan-3-one 33

As for general procedure D. A 2:1 mixture of (E)-3-(4-oxo-hexylidene)-tetrahydropyran-2-one $\mathbf{2 2 E}$ and (Z)-3-(4-oxo-hexylidene)-tetrahydro-pyran-2-one $\mathbf{2 2 Z}$ (40 $\mathrm{mg}$, $0.20 \mathrm{mmol}, 1 \mathrm{eq}$ ), after reaction time of $1 \mathrm{~h}$ and purification of the crude product mixture by column chromatography (eluting with $80 \%$ EtOAc in petroleum ether (40$\left.\left.60^{\circ} \mathrm{C}\right)\right)$ gave rel-(1R, 4R/S, 5R)-1-ethyl-4-(3-hydroxy-propyl)-2-oxabicyclo[3.2.0]heptan-3-one $\mathbf{3 3}$ (20 $\mathrm{mg}, 0.10 \mathrm{mmol}, 50 \%)$ as a clear, colourless oil:

\section{Characterised as a mixture of diastereoisomers}

$\square_{\max }($ neat $) / \mathrm{cm}^{-1} 3413 \mathrm{bs}(\mathrm{OH}), 2939 \mathrm{~m}, 1747 \mathrm{~s}(\mathrm{C}=\mathrm{O}), 1460 \mathrm{~m}, 1207 \mathrm{~m}, 1055 \mathrm{~m}, 1022 \mathrm{~m}$ and $941 \mathrm{~m} ; \square_{\mathrm{H}}\left(400 \mathrm{MHz}, \mathrm{CDCl}_{3}\right) 0.85\left(3 \mathrm{H}, \mathrm{t}, J 7.4, \mathrm{CH}_{3}\right.$ from minor), $0.88(3 \mathrm{H}, \mathrm{t}, J$ 7.4, $\mathrm{CH}_{3}$ from major), $1.45-2.17\left(20 \mathrm{H}, \mathrm{m}, 5 \times \mathrm{CH}_{2}\right.$ from minor, $5 \mathrm{x} \mathrm{CH}_{2}$ from major), $2.42-2.46(1 \mathrm{H}, \mathrm{m}, \mathrm{CHCHC}(\mathrm{O})$ from major $), 2.52-2.56(1 \mathrm{H}, \mathrm{m}, \mathrm{CHCHC}(\mathrm{O})$ from major), $2.61-2.68(1 \mathrm{H}, \mathrm{m}, \mathrm{CHCHC}(\mathrm{O})$ from minor), $2.90(1 \mathrm{H}$, apparent q, $J$ 8.0, $\mathrm{CHCHC}(\mathrm{O})$ from minor) and $3.59-3.62\left(4 \mathrm{H}, \mathrm{m}, \mathrm{CH}_{2} \mathrm{OH}\right.$ from minor, $\mathrm{CH}_{2} \mathrm{OH}$ from major); $\mathrm{Z}_{\mathrm{c}}\left(100 \mathrm{MHz}, \mathrm{CDCl}_{3}\right) 7.9\left(\mathrm{CH}_{3}\right.$ from minor $), 8.1\left(\mathrm{CH}_{3}\right.$ from major), 16.3 $\left(\mathrm{CH}_{2}\right), 21.7\left(\mathrm{CH}_{2}\right), 22.6\left(\mathrm{CH}_{2}\right), 29.0\left(\mathrm{CH}_{2}\right), 30.3\left(\mathrm{CH}_{2}\right), 30.4\left(\mathrm{CH}_{2}\right), 30.6\left(\mathrm{CH}_{2}\right), 30.7$ $\left(\mathrm{CH}_{2}\right), 31.5\left(\mathrm{CH}_{2}\right), 31.6\left(\mathrm{CH}_{2}\right), 41.1(\mathrm{CHCHC}(\mathrm{O})$ from minor), 43.1 ( $\mathrm{CHCHC}(\mathrm{O})$ from major), $44.4(\mathrm{CHCHC}(\mathrm{O})$ from minor), $49.5(\mathrm{CHCHC}(\mathrm{O})$ from major), 62.7 ( $\mathrm{CH}_{2} \mathrm{OH}$ from major), $62.8\left(\mathrm{CH}_{2} \mathrm{OH}\right.$ from minor $), 89.7\left(\mathrm{C}\left(\mathrm{CH}_{2} \mathrm{CH}_{3}\right) \mathrm{O}\right.$ from major $)$, $90.4\left(\mathrm{C}\left(\mathrm{CH}_{2} \mathrm{CH}_{3}\right) \mathrm{O}\right.$ from minor), $179.8(\mathrm{C}(\mathrm{O})$ from minor) and $180.9(\mathrm{C}(\mathrm{O})$ from major); $m / z$ (CI mode, isobutane) $200(10 \%), 199\left((\mathrm{M}+\mathrm{H})^{+} 100\right), 181(10), 169$ (2), 
129 (5), 115 (2), 85 (10) and 71 (10); (Found: $(\mathrm{M}+\mathrm{H})^{+}$, 199.1335. $\mathrm{C}_{11} \mathrm{H}_{19} \mathrm{O}_{3}$ requires $M, 199.1334)$.

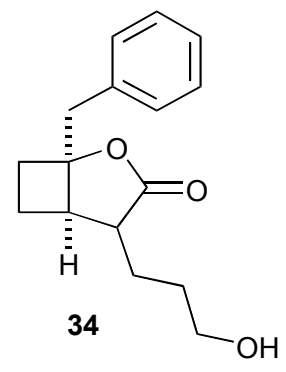

rel-(1S, 4R/S, 5R)-1-Benzyl-4-(3-hydroxy-propyl)-2-oxabicyclo[3.2.0]heptan-3-one 34

As for general procedure D. (E)-3-(4-Oxo-5-phenyl-pentylidene)-tetrahydro-pyran-2one $23 E$ ( $20 \mathrm{mg}, 0.08 \mathrm{mmol}, 1 \mathrm{eq}$ ), after reaction time of $2.5 \mathrm{~h}$ and purification of the crude product mixture by column chromatography (eluting with $80 \%$ EtOAc in petroleum ether $\left(40-60^{\circ} \mathrm{C}\right)$ ) gave $\mathrm{rel}-(1 \mathrm{~S}, 4 \mathrm{R} / \mathrm{S}, 5 \mathrm{R})$-1-benzyl-4-(3-hydroxy-propyl)-2oxa-bicyclo[3.2.0]heptan-3-one 34 (7 $\mathrm{mg}, 0.03 \mathrm{mmol}, 34 \%)$ as a clear, colourless oil:

\section{Characterised as a mixture of diastereoisomers}

$\square_{\max }($ neat $) / \mathrm{cm}^{-1} 3408 \mathrm{bs}(\mathrm{OH}), 2941 \mathrm{~m}, 1747 \mathrm{~s}(\mathrm{C}=\mathrm{O}), 1454 \mathrm{~m}, 1261 \mathrm{~m}, 1171 \mathrm{~m}, 1053 \mathrm{~m}$ and 947; $\square_{\mathrm{H}}\left(400 \mathrm{MHz}, \mathrm{CDCl}_{3}\right) 0.60-0.70\left(1 \mathrm{H}, \mathrm{m}, 1 \mathrm{H}\right.$ from $\mathrm{CH}_{2}$ from major $), 1.13-$ $1.20\left(1 \mathrm{H}, \mathrm{m}, 1 \mathrm{H}\right.$ from $\mathrm{CH}_{2}$ from major), $1.30-1.99\left(9 \mathrm{H}, \mathrm{m}, \mathrm{CH}\right.$ from minor, $2 \times \mathrm{CH}_{2}$ from minor, $2 \mathrm{x} \mathrm{CH}_{2}$ from major), $2.12-2.26\left(4 \mathrm{H}, \mathrm{m}, \mathrm{CH}_{2}\right.$ from minor, $\mathrm{CH}_{2}$ from major), $2.34-2.40$ (1H, m, $\mathrm{CH}$ from major), $2.49-2.55$ (1H, m, $\mathrm{CH}$ from major), $2.82\left(1 \mathrm{H}, \mathrm{d}, J 14.1, \mathrm{AB}\right.$ system, $1 \mathrm{H}$ from $\mathrm{CH}_{2} \mathrm{Ph}$ from minor), $2.83(1 \mathrm{H}, \mathrm{d}, J 14.2, \mathrm{AB}$ system, $1 \mathrm{H}$ from $\mathrm{CH}_{2} \mathrm{Ph}$ from major), $2.95(1 \mathrm{H}$, apparent q, $J$ 7.8, $\mathrm{CH}$ from minor), $3.03\left(1 \mathrm{H}, \mathrm{d}, J 14.1, \mathrm{AB}\right.$ system, $1 \mathrm{H}$ from $\mathrm{CH}_{2} \mathrm{Ph}$ from minor), $3.09(1 \mathrm{H}, \mathrm{d}, J 14.2, \mathrm{AB}$ system, $1 \mathrm{H}$ from $\mathrm{CH}_{2} \mathrm{Ph}$ from major), 3.35 - 3.45 (2H, m, $\mathrm{CH}_{2} \mathrm{OH}$ from major), $3.46-$ $3.52\left(2 \mathrm{H}, \mathrm{m}, \mathrm{CH}_{2} \mathrm{OH}\right.$ from minor) and $7.13-7.29(10 \mathrm{H}, \mathrm{m}, \mathrm{Ar} \mathrm{CH}$ from minor, $\mathrm{Ar}$ $\mathrm{CH}$ from major); $\mathrm{Z}_{\mathrm{C}}\left(100 \mathrm{MHz}, \mathrm{CDCl}_{3}\right) 15.3\left(\mathrm{CH}_{2}\right), 20.4\left(\mathrm{CH}_{2}\right), 20.9\left(\mathrm{CH}_{2}\right), 26.4$ $\left(\mathrm{CH}_{2}\right), 29.2\left(\mathrm{CH}_{2}\right), 29.8\left(\mathrm{CH}_{2}\right), 30.0\left(\mathrm{CH}_{2}\right), 31.0\left(\mathrm{CH}_{2}\right), 39.8(\mathrm{CH}$ from minor $), 40.7$ ( $\mathrm{CH}$ from major), $42.2\left(\mathrm{CH}_{2} \mathrm{Ph}\right.$ from minor), $42.3\left(\mathrm{CH}_{2} \mathrm{Ph}\right.$ from major), 42.7 ( $\mathrm{CH}$ 
from minor), 48.1 ( $C \mathrm{H}$ from major), $61.2\left(\mathrm{CH}_{2} \mathrm{OH}\right.$ from major), $61.3\left(\mathrm{CH}_{2} \mathrm{OH}\right.$ from minor), $87.7\left(C\left(\mathrm{CH}_{2} \mathrm{Ph}\right) \mathrm{O}\right.$ from major), $87.8\left(\mathrm{C}\left(\mathrm{CH}_{2} \mathrm{Ph}\right) \mathrm{O}\right.$ from minor), $125.9(\mathrm{Ar} \mathrm{CH}$ from minor), 126.0 (Ar $\mathrm{CH}$ from major), 127.4 (2 x $\mathrm{Ar} C \mathrm{H}$ from major), 127.5 (2 x $\mathrm{Ar}$ $\mathrm{CH}$ from minor), 128.8 (2 x $\mathrm{Ar} \mathrm{CH}$ from minor), 129.2 (4 (2 x $\mathrm{Ar} \mathrm{CH}$ from major), 134.3 (Ar $C$ from minor), 134.5 (Ar $C$ from major), $178.2(C(\mathrm{O})$ from minor) and 179.2 ( $C(\mathrm{O})$ from major); $m / z$ (EI mode) $260\left(\left(\mathrm{M}^{+\bullet}\right)\right.$ 10\%), 232 (5), $214(20), 186$ (20), 169 (70), 141 (40), 123 (100), 91 (80), 81 (40), 67 (20) and 41 (15); (Found: $\left(\mathrm{M}^{+\bullet}\right), 260.1412 . \mathrm{C}_{16} \mathrm{H}_{20} \mathrm{O}_{3}$ requires $\left.M, 260.1412\right)$.

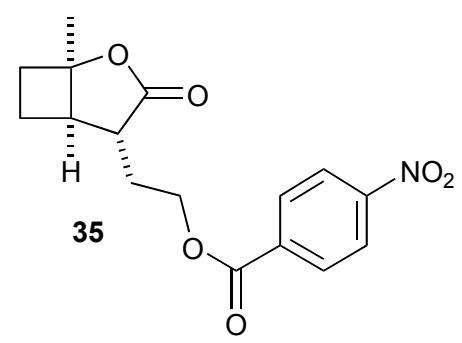

\section{rel-(1R, 4R, 5R)-Ethyl-2-(1-methyl-3-oxo-2-oxa-bicyclo[3.2.0]hept-4-yl)-4- nitrobenzoate 35}

4-Nitrobenzoyl chloride (46 mg, $0.25 \mathrm{mmol}, 2 \mathrm{eq}$ ) was added to a stirred solution of 4-(2-hydroxy-ethyl)-1-methyl-2-oxa-bicyclo[3.2.0]heptan-3-one 30 (21 mg, 0.12 mmol, 1 eq) in pyridine $(1 \mathrm{ml})$ at room temperature. The solution was stirred for $2 \mathrm{~h}$ before addition of $\mathrm{H}_{2} \mathrm{O}$ (dist.) $(1 \mathrm{ml})$. The aqueous layer was separated and extracted with EtOAc ( 4 x $10 \mathrm{ml})$. The combined organic extracts were dried $\left(\mathrm{MgSO}_{4}\right)$, and concentrated in vacuo to give the crude product. Purification by column chromatography (eluting with $30 \% \mathrm{Et}_{2} \mathrm{O}$ in $\mathrm{CHCl}_{3}$ ) gave rel-(1R, 4R, 5R)-ethyl-2-(1methyl-3-oxo-2-oxa-bicyclo[3.2.0]hept-4-yl)-4-nitrobenzoate 35 (20 mg, 0.06 mmol, $51 \%$ ) as white crystals: $\mathrm{mp} 130-133^{\circ} \mathrm{C}$ (in EtOH): $\square_{\max }\left(\right.$ golden gate) $/ \mathrm{cm}^{-1} 2954 \mathrm{w}$, 1743s (lactone $\mathrm{C}=\mathrm{O}$ ), 1724s (ester $\mathrm{C}=\mathrm{O}$ ), 1520s, 1344m, 1269s, 1236s, 1119s, 1103s, $1011 \mathrm{~m}, 958 \mathrm{~s}, 877 \mathrm{~s}$ and $839 \mathrm{~s} ; \square_{\mathrm{H}}\left(400 \mathrm{MHz} \mathrm{CDCl}_{3}\right) 1.43\left(3 \mathrm{H}, \mathrm{s}, \mathrm{CH}_{3}\right), 1.63-1.68$ $\left(1 \mathrm{H}, \mathrm{m}, 1 \mathrm{H}\right.$ from $\left.\mathrm{CCH}_{2} \mathrm{CH}_{2} \mathrm{CH}\right), 1.93-2.02\left(1 \mathrm{H}, \mathrm{m}, 1 \mathrm{H}\right.$ from $\left.\mathrm{CH}_{2} \mathrm{CH}_{2} \mathrm{O}\right), 2.10-2.28$ $\left(4 \mathrm{H}, \mathrm{m}, 3 \mathrm{H}\right.$ from $\mathrm{CCH}_{2} \mathrm{CH}_{2} \mathrm{CH}, 1 \mathrm{H}$ from $\left.\mathrm{CH}_{2} \mathrm{CH}_{2} \mathrm{O}\right), 2.51-2.56(1 \mathrm{H}, \mathrm{m}$, $\mathrm{CHCHC}(\mathrm{O})), 2.70(1 \mathrm{H}, \mathrm{dt}, J 3.0,6.1, \mathrm{CHCHC}(\mathrm{O})), 4.37-4.48\left(2 \mathrm{H}, \mathrm{m}, \mathrm{CH}_{2} \mathrm{O}\right), 8.14$ (2H, apparent dt, $J 1.9,8.9,2 \times \mathrm{Ar} \mathrm{CH}$ ) and 8.23 (2H, apparent dt, $J 1.9,8.9,2 \times \mathrm{Ar}$ 
$\mathrm{CH}) ; \square_{\mathrm{C}}\left(100 \mathrm{MHz}, \mathrm{CDCl}_{3}\right) 21.4\left(1 \mathrm{C}\right.$ from $\left.\mathrm{CCH}_{2} \mathrm{CH}_{2} \mathrm{CH}\right), 24.7\left(\mathrm{CH}_{3}\right), 31.3$ $\left(\mathrm{CH}_{2} \mathrm{CH}_{2} \mathrm{O}\right), 33.6$ (1C from $\left.\mathrm{CCH}_{2} \mathrm{CH}_{2} \mathrm{CH}\right), 44.6(\mathrm{CHCHC}(\mathrm{O})), 46.9(\mathrm{CHC}(\mathrm{O})), 63.6$

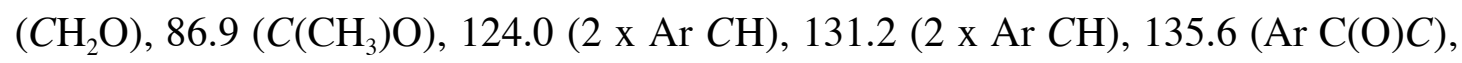
151.1 ( $\left.\mathrm{Ar} C \mathrm{NO}_{2}\right), 164.9(\mathrm{Ar} C(\mathrm{O}))$ and $179.7(C(\mathrm{O}) \mathrm{O}) ; \mathrm{m} / \mathrm{z}(\mathrm{CI}$ mode, isobutane) 320 $\left((\mathrm{M}+\mathrm{H})^{+}\right.$100), 290 (50), 171 (15), 153 (10), 138 (5), 120 (25), 113 (5), 97 (5), 85 (5) and 71 (10); (Found: $(\mathrm{M}+\mathrm{H})^{+}, 320.1135 . \mathrm{C}_{16} \mathrm{H}_{18} \mathrm{O}_{6} \mathrm{~N}$ requires $\left.M, 320.1134\right)$.

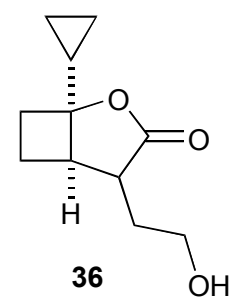

\section{rel-(1S, 4R/S， 5R)-1-Cyclopropyl-4-(2-hydroxy-ethyl)-2-oxa- bicyclo[3.2.0]heptan-3-one 36}

As for general procedure E. (E)-3-(4-Oxo-4-cyclopropyl-butylidene)-dihydro-furan-2one 10 (50 $\mathrm{mg}, 0.26 \mathrm{mmol}, 1 \mathrm{eq})$, after reaction time of $2.5 \mathrm{~h}$ and purification of the crude product mixture by column chromatography (eluting with $50 \%$ EtOAc in petroleum ether $\left(40-60^{\circ} \mathrm{C}\right)$, then with $30 \% \mathrm{Et}_{2} \mathrm{O}$ in $\left.\mathrm{CHCl}_{3}\right)$ gave $\mathrm{rel}-(1 \mathrm{~S}, 4 \mathrm{R} / \mathrm{S}, 5 \mathrm{R})-1$ cyclopropyl-4-(2-hydroxy-ethyl)-2-oxa-bicyclo[3.2.0]heptan-3-one 36 (36 mg, 0.18 mmol, $70 \%$ ) as a clear, colourless oil:

\section{Characterised as a mixture of diastereoisomers}

$\square_{\max }($ neat $) / \mathrm{cm}^{-1} 3413 \mathrm{bs}(\mathrm{OH}), 2943 \mathrm{~m}, 1743 \mathrm{~s}(\mathrm{C}=\mathrm{O}), 1238 \mathrm{~m}, 1167 \mathrm{~m}, 1051 \mathrm{~m}$ and 945m; $\square_{\mathrm{H}}\left(400 \mathrm{MHz}, \mathrm{CDCl}_{3}\right) 0.28-0.32\left(4 \mathrm{H}, \mathrm{m}, \mathrm{OCCH}\left(\mathrm{CH}_{2}\right)_{2}{ }^{\mathrm{A}}\right.$ from minor, $\mathrm{OCCH}\left(\mathrm{CH}_{2}\right)_{2}{ }^{\mathrm{A}}$ from major), $0.46-0.52\left(4 \mathrm{H}, \mathrm{m}, \mathrm{OCCH}\left(\mathrm{CH}_{2}\right)_{2}{ }^{\mathrm{B}}\right.$ from minor, $\mathrm{OCCH}\left(\mathrm{CH}_{2}\right)_{2}{ }^{\mathrm{B}}$ from major), $1.02-1.10\left(2 \mathrm{H}, \mathrm{m}, \mathrm{OCCH}\left(\mathrm{CH}_{2}\right)_{2}\right.$ from minor, $\mathrm{OCCH}\left(\mathrm{CH}_{2}\right)_{2}$ from major), $1.62-2.12\left(12 \mathrm{H}, \mathrm{m}, 3 \times \mathrm{CH}_{2}\right.$ from minor, $3 \times \mathrm{CH}_{2}$ from major), $2.42-2.46(1 \mathrm{H}, \mathrm{m}, \mathrm{CH}$ from major), $2.67-2.71$ (1H, m, $\mathrm{CH}$ from major), $2.81-2.88\left(2 \mathrm{H}, \mathrm{m}, 2 \times \mathrm{CH}\right.$ from minor), $3.57-3.62\left(1 \mathrm{H}, \mathrm{m}, 1 \mathrm{H}\right.$ from $\mathrm{CH}_{2} \mathrm{OH}$ from minor) and $3.68-3.78\left(3 \mathrm{H}, \mathrm{m}, 1 \mathrm{H}\right.$ from $\mathrm{CH}_{2} \mathrm{OH}$ from minor, $\mathrm{CH}_{2} \mathrm{OH}$ from major); $\square_{\mathrm{c}}$ $\left(100 \mathrm{MHz}, \mathrm{CDCl}_{3}\right)-0.4\left(\mathrm{OCH}\left(\mathrm{CH}_{2}\right)_{2}{ }^{\mathrm{AB}}\right),-0.3\left(\mathrm{OCH}\left(\mathrm{CH}_{2}\right)_{2}{ }^{\mathrm{AB}}\right),-0.1\left(\mathrm{OCH}\left(\mathrm{CH}_{2}\right)_{2}{ }^{\mathrm{AB}}\right)$, 
$0.0\left(\mathrm{OCH}\left(\mathrm{CH}_{2}\right)_{2}{ }^{\mathrm{AB}}\right), 14.0\left(\mathrm{OCH}\left(\mathrm{CH}_{2}\right)_{2}\right), 14.6\left(\mathrm{OCH}\left(\mathrm{CH}_{2}\right)_{2}\right), 15.1\left(\mathrm{CH}_{2}\right), 20.0\left(\mathrm{CH}_{2}\right)$, $27.2\left(\mathrm{CH}_{2}\right), 27.5\left(\mathrm{CH}_{2}\right), 28.4\left(\mathrm{CH}_{2}\right), 33.4\left(\mathrm{CH}_{2}\right), 39.1(\mathrm{CH}), 40.7(\mathrm{CH}), 41.4(\mathrm{CH})$, $45.1(\mathrm{CH}), 59.1\left(\mathrm{CH}_{2} \mathrm{OH}\right), 59.8\left(\mathrm{CH}_{2} \mathrm{OH}\right), 88.4(\mathrm{CCH}), 89.1(\mathrm{CCH}), 178.3(\mathrm{C}(\mathrm{O}))$ and $179.3(C(\mathrm{O})) ; \mathrm{m} / \mathrm{z}\left(\mathrm{CI}\right.$ mode, isobutane) $197\left((\mathrm{M}+\mathrm{H})^{+} 100\right), 179$ (10), 169 (1), 151(2), 135 (2), 15 (5), 93 (2), 85 (2) and 71 (2); (Found: $(\mathrm{M}+\mathrm{H})^{+}, 197.1177$. $\mathrm{C}_{11} \mathrm{H}_{17} \mathrm{O}_{3}$ requires $M, 197.1178$ ). 